JOURNAL OF THE

AMERICAN MATHEMATICAL SOCIETY

Volume 23, Number 1, January 2010, Pages 189-215

S 0894-0347(09)00641-9

Article electronically published on July 31,2009

\title{
CONSTRUCTING WEYL GROUP MULTIPLE DIRICHLET SERIES
}

\author{
GAUTAM CHINTA AND PAUL E. GUNNELLS
}

\section{INTRODUCTION}

This paper describes a technique to construct Weyl group multiple Dirichlet series. Such series were first introduced in $\mathrm{BBC}^{+} 06$, which also described a heuristic means to define, analytically continue, and prove functional equations for a family of Dirichlet series in several complex variables. Several subsequent papers have dealt with the problem of making the heuristic definitions precise and completing the proofs of analytic continuation and functional equations of these Weyl group multiple Dirichlet series along the lines suggested in $\left[\mathrm{BBC}^{+} 06\right.$. . Before listing some of the partial results obtained in these papers, we say a bit more about the type of multiple Dirichlet series studied.

Let $F$ be an algebraic number field containing the $2 n$th roots of unity. Fix a finite set of places $S$ containing all the Archimedean places and those that are ramified over $\mathbb{Q}$. Take $S$ large enough that $\mathcal{O}_{S}$, the ring of $S$-integers of $F$, has class number one.

Let $\Phi$ be a reduced root system of rank $r$. Let $\mathbf{m}=\left(m_{1}, \ldots, m_{r}\right)$ be an $r$ tuple of integers in $\mathcal{O}_{S}$ and $\mathbf{s}=\left(s_{1}, \ldots, s_{n}\right)$ an $r$-tuple of complex variables. In (5.6) we define a certain finite-dimensional vector space $\mathcal{M}(\Omega, \Phi)$ of complex-valued functions on $\left(F_{S}^{\times}\right)^{r}$, and we choose $\Psi \in \mathcal{M}(\Omega, \Phi)$. In Section 4 we define coefficients $H(\mathbf{c} ; \mathbf{m})$, where $\mathbf{c}$ and $\mathbf{m}$ are $r$-tuples of nonzero integers in $\mathcal{O}_{S}$. To this data we associate a multiple Dirichlet series in $r$ complex variables

$$
Z(\mathbf{s} ; \mathbf{m}, \Psi)=Z(\mathbf{s} ; \mathbf{m}, \Psi ; \Phi, n)=\sum_{\mathbf{c}} \frac{H(\mathbf{c} ; \mathbf{m}) \Psi(\mathbf{c})}{\prod\left|c_{i}\right|^{s_{i}}}
$$

where $\mathbf{c}=\left(c_{1}, \ldots, c_{r}\right)$ and each $c_{i}$ ranges over $\left(\mathcal{O}_{S} \backslash\{0\}\right) / \mathcal{O}_{S}^{\times}$. (Although the coefficients $H$ and $\Psi$ are defined for nonzero $c_{i} \in \mathcal{O}_{S}$, their product $H \Psi$ is unchanged when any $c_{i}$ is multiplied by a unit, as the arguments in BBF06 show.) The sum (1.1) is absolutely convergent when $\operatorname{Re}\left(s_{i}\right)>3 / 2$. We call (1.1) a degree $n$ Weyl group multiple Dirichlet series of type $\Phi$ with twisting parameter $\mathbf{m}$.

Received by the editors March 11, 2008.

2000 Mathematics Subject Classification. Primary 11F66, 11M41; Secondary 11F37, 11F70, $22 \mathrm{E} 99$.

Key words and phrases. Weyl group multiple Dirichlet series, Fourier coefficients of Eisenstein series, Weyl character formula, metaplectic groups.

Both authors thank the NSF for support.

(C)2009 American Mathematical Society Reverts to public domain 28 years from publication 
These series were introduced in the papers $\mathrm{BBC}^{+} 06$, BBFH07, BBF08. Actually, except for the cases listed below, in these papers the coefficients

$$
H\left(c_{1}, \ldots, c_{r} ; m_{1}, \ldots, m_{r}\right)
$$

were defined only when the product $c_{1} \cdots c_{r} \cdot m_{1} \cdots m_{r}$ was squarefree (we call these the powerfree coefficients), and it was suggested that it should be possible to define the $H$-coefficients for all $r$-tuples $\left(c_{1}, \ldots, c_{r}\right)$ in such a way that the resulting multiple Dirichlet series has an analytic continuation to $\mathbb{C}^{r}$ and satisfies a group of functional equations isomorphic to the Weyl group $W$ of $\Phi$. These powerfree coefficients can be expressed in terms of $n$ th-order Gauss sums and the root data.

The full definitions and expected properties of $Z(\mathbf{s} ; \mathbf{m}, \Psi ; \Phi, n)$ have been given in the following cases:

(1) $\left.\mathrm{BBC}^{+} 06\right] \Phi=A_{2}, n$ arbitrary, $\mathbf{m}=(1,1)$.

(2) BBF06, BBF08 $\Phi$ arbitrary and the degree $n$ sufficiently large with respect to $\Phi$ and the twisting parameter $\mathbf{m}$. In $\mathrm{BBF} 06, \overline{\mathrm{BBF} 08}$, this is called the stable case.

(3) BBFH07 $\Phi=A_{2}, n$ arbitrary, $\mathbf{m}$ arbitrary.

(4) CG07, CFG08 $\Phi$ simply-laced, $n=2$, i.e. the quadratic case. (The methods of [CG07, which dealt only with the untwisted case, were extended to arbitrary $\mathbf{m}$ in CFG08.)

(5) $[\mathrm{BBFa}, \mathrm{BBFb}] \Phi=A_{r}, n$ arbitrary, $\mathbf{m}$ arbitrary. In these preprints, Brubaker, Bump and Friedberg deal with the type $A_{r}$ multiple Dirichlet series in two different ways. In $[\mathrm{BBF}]$ they show that the multiple Dirichlet series coincide with Whittaker coefficients of a metaplectic Eisenstein series, while in $\mathrm{BBFb}$, they use a combinatorial approach based on crystal graphs. At present, both methods work only for the type $A$ root systems.

In this paper we give, by a uniform method, a complete definition of the Weyl group multiple Dirichlet series and proofs of the expected properties in all cases. That is, we have the following theorem (cf. Theorem 6.1):

Theorem 1.1. Let $n \geq 1$ and let $F, S, \mathcal{O}_{S}$ be as above. Let $\Phi$ be a reduced root system of rank $r$ and let $\mathbf{m}$ be an $r$-tuple of nonzero integers in $\mathcal{O}_{S}$. Then, the multiple Dirichlet series $Z(\mathbf{s} ; \mathbf{m}, \Psi ; \Phi, n)$ defined in Section 6 has an analytic continuation to $\mathbf{s} \in \mathbb{C}^{r}$ and satisfies a group of functional equations isomorphic to $W$, the Weyl group of $\Phi$.

We now describe our method of proof. As stated above, the heuristic definition of $Z(\mathbf{s} ; \mathbf{m}, \Psi)$ includes the definition of the powerfree coefficients $H\left(c_{1}, \ldots, c_{r} ; m_{1}, \ldots\right.$, $m_{r}$ ). Moreover, the coefficients satisfy a twisted multiplicativity (4.3)-4.5) that reduces their specification to that of the prime power coefficients $H\left(\varpi^{k_{1}}, \ldots, \varpi^{k_{r}}\right.$; $\left.\varpi^{l_{1}}, \ldots, \varpi^{l_{r}}\right)$, for $\varpi$ prime in $\mathcal{O}_{S}$. We are naturally led to consider the generating series

$$
N=N\left(x_{1}, \ldots, x_{r}\right)=\sum_{k_{1}, \ldots, k_{r} \geq 0} H\left(\varpi^{k_{1}}, \ldots, \varpi^{k_{r}}\right) x_{1}^{k_{1}} \cdots x_{r}^{k_{r}}
$$

where we have suppressed the twisting parameter from the notation. This generating series, which turns out to be a polynomial in the indeterminates $x_{1}, \ldots, x_{r}$, must be defined in such a way that the resulting global object $Z(\mathbf{s} ; \mathbf{m}, \Psi)$ can be shown to satisfy the right functional equations. 
The main idea of this paper is that there is a certain action of $W$ on the field of rational functions $\mathbb{C}\left(x_{1}, \ldots, x_{r}\right)$. Using this action we can construct an invariant function $h$ with an easily understood denominator $D \in \mathbb{C}\left[x_{1}, \ldots, x_{r}\right]$. We then take $N$ to be the polynomial $h D$, and we prove that the multiple Dirichlet series constructed using (1.2) satisfies the requisite functional equations under $W$. What makes things difficult is that the $W$-action is a little complicated. Moreover, it is not obvious that this action has anything to do with the functional equations of a global multiple Dirichlet series.

There are at least three ways to motivate the $W$-action defined in Section 3 , First, if the functional equations of $Z(\mathbf{s} ; \mathbf{m}, \Psi)$ are assumed, and one works backwards through the proof of Theorem [5.8, one finds that the $\varpi$-parts of the multiple Dirichlet series must have precisely the $W$-invariance defined in Section3. For $n=2$ and $\Phi=A_{2}$, this is the approach taken in Section 5 of [CFH06. A second way to motivate the action of $W$ is the remarkable fact that an untwisted Weyl group multiple Dirichlet series over the rational function field coincides (after a simple change of variables) with its own $\varpi$-part. This was noted in [CFH06] for $\Phi=A_{2}$ and $n=2$ and in Chi08 for $\Phi=A_{2}$ and general $n$. Consequently, in this context the $\varpi$-parts satisfy essentially the same functional equations as the multiple Dirichlet series itself. These functional equations follow from the functional equations of Kubota's Dirichlet series. Over the rational function field, Kubota's theory has been very explicitly worked out by Hoffstein Hof92 and Patterson Pat07. This is the point of view which motivated our work in $\overline{C G}$. Yet another approach to motivating the local functional equations is via the Eisenstein conjecture described in BBF06. This conjecture essentially states that a Weyl group multiple Dirichlet series should be a Whittaker coefficient of an Eisenstein series on an $n$-fold metaplectic cover of $G$, the simply-connected algebraic group over $F$ whose root system is the dual of $\Phi$. If this is true, then the $\varpi$-part of a multiple Dirichlet series should be related to a local Whittaker function of a $\varpi$-adic metaplectic group. The functional equations satisfied by a $\varpi$-adic metaplectic Whittaker function have been described by Kazhdan-Patterson KP84. At least for root systems of type $A$, the group action we define in Section 3 coincides with the functional equations in Lemma 1.3.3 of KP84. From this perspective, our averaging formula (3.25) can potentially be seen as a Casselman-Shalika formula for $\varpi$-adic Whittaker coefficients on metaplectic groups. This connection is being further developed in current work of Chinta and Offen.

We conclude this introduction with a description of the organization of the paper. In Section 2 we review basic facts about Gauss sums and reciprocity that we will need. Section 3 is devoted to the definition of the action of the Weyl group on the field of rational functions $\mathbb{C}\left(x_{1}, \ldots, x_{r}\right)$. We further construct rational functions invariant under the group action. These invariant functions play a key role in the definition of the Weyl group multiple Dirichlet series. The results of this section depend only on the combinatorics of the root system and its Weyl group.

Section 4 describes how to define the coefficients $H(\mathbf{c} ; \mathbf{m})$ of the multiple Dirichlet series. In short, the prime power coefficients

$$
H\left(\varpi^{\beta_{1}}, \ldots, \varpi^{\beta_{r}} ; \varpi^{l_{1}}, \ldots, \varpi^{l_{r}}\right)
$$

can be read off from the Taylor coefficients of the invariant functions constructed in Section 3 The general coefficient $H(\mathbf{c} ; \mathbf{m})$ is then defined in terms of the prime power coefficients via the "twisted multiplicativity" given in (4.3). In addition we 
show that certain combinations $f^{\varpi ; \mathbf{k}}$ of the local factors satisfy the simple functional equation given in Theorem 4.1 .

Section 5 begins with a review of Kubota's Gauss sum Dirichlet series, as presented in BB06a. These Dirichlet series, constructed with Gauss sum coefficients, have a meromorphic continuation to the complex plane and satisfy a functional equation as $s \mapsto 2-s$; see Proposition 5.1. We further construct a single-variable Dirichlet series $\mathcal{E}$ (defined in (5.9) using the coefficients $H(\mathbf{c} ; \mathbf{m})$, and show that it can be written as a linear combination of products of Kubota's series and the local factors $f^{\varpi ; \mathbf{k}}$ defined in Section 4 . Consequently, $\mathcal{E}$ satisfies the functional equation described in Theorem 5.8 .

In Section 6 we define the multiple Dirichlet series $Z(\mathbf{s} ; \mathbf{m}, \Psi)$ and prove our main result, Theorem 6.1, which gives the functional equation and meromorphic continuation of $Z$. The single-variable Dirichlet series $\mathcal{E}$ act as the building blocks of $Z$, and hence the functional equations of the multivariable object follow from those of $\mathcal{E}$ established in the previous section. Then the meromorphic continuation of the multiple Dirichlet series $Z$ follows from a simple convexity argument, as has been extensively used in the recent papers [CG07, $\mathrm{BBFH07}, \mathrm{BBC}^{+} 06, \mathrm{BBF} 06, \mathrm{CG}$.

In Section 7 we suggest how other examples of multiple Dirichlet series that have previously appeared in the literature fall into the framework presented here, and we make some further remarks. This final section is not meant to be absolutely precise or definitive, but rather indicative of further prospects for research in the field.

As the outline given above suggests, we have tried to minimize the interdependence of the sections on one another. In particular the only result from Section 4 that is used in the later sections is Theorem 4.1, which appears at the very end of the proof of Theorem 5.8. Theorem 5.8 is then the only theorem from the earlier sections used in the proof of our main result, Theorem 6.1. The structure of this argument mimics the pattern established in our earlier papers [CG07, Chi05, CG], where functional equations of local factors induce functional equations in certain univariate Dirichlet series, which in turn induce functional equations in the multivariate Weyl group multiple Dirichlet series that are our main objects of interest. This approach has by now become streamlined and dense, as necessitated by the increasing notational complexities. The reader who is new to these techniques may benefit from the presentation in [CG], where the case of the root system $A_{2}$ is worked out in detail.

\section{Preliminaries}

In this section we recall basic facts about Hilbert symbols and Gauss sums that we will need in the sequel. Our exposition follows [BBF08, $§ \S 2.3-2.7$ ] essentially verbatim.

Recall that $F$ is a number field containing the $2 n$th roots of unity, and that $S$ is a finite set of places containing all the Archimedean places and those ramified over $\mathbb{Q}$. Recall also that $S$ is assumed to be large enough that $\mathcal{O}_{S}$, the ring of $S$-integers of $F$, is a principal ideal domain. Let $F_{S}=\prod_{v \in S} F_{v}$. Similarly let $S_{\text {fin }} \subset S$ be the subset of finite places, and let $F_{\text {fin }}=\prod_{v \in S_{\text {fin }}} F_{v}$. We embed $\mathcal{O}_{S} \rightarrow F_{S}$ diagonally.

Each place $v$ determines a local Hilbert symbol $(,)_{v}: F_{v}^{\times} \times F_{v}^{\times} \rightarrow \mu_{n}$, where $\mu_{n}$ is the group of $n$th roots of unity Neu99. We assume fixed an embedding $\epsilon$ from 
$\mu_{n}$ to $\mathbb{C}^{\times}$and identify $\mu_{n}$ with its image. The Hilbert symbol leads to a pairing

$$
(,)_{S}: F_{S}^{\times} \times F_{S}^{\times} \rightarrow \mu_{n}
$$

defined by $(a, b)_{S}=\prod_{v \in S}(a, b)_{v}$.

A subgroup $\Omega \subset F_{S}^{\times}$is called isotropic if $\left.(,)_{S}\right|_{\Omega \times \Omega}$ is trivial. Let $\Omega$ be the subgroup $\mathcal{O}_{S}^{\times} F_{S}^{\times n}$, which is maximal isotropic. Given a positive integer $t$, define the complex vector space $\mathcal{M}_{t}(\Omega)$ by

$$
\mathcal{M}_{t}(\Omega)=\left\{\Psi: F_{\text {fin }}^{\times} \rightarrow \mathbb{C} \mid \Psi(\varepsilon c)=(c, \varepsilon)_{S}^{-t} \Psi(c) \text { for all } \varepsilon \in \Omega\right\} .
$$

We abbreviate $\mathcal{M}_{1}(\Omega)$ by $\mathcal{M}(\Omega)$. Note that if $\varepsilon$ is sufficiently close to the identity in $F_{\text {fin }}^{\times}$, then $\varepsilon$ is an $n$th power at every finite place $S_{\text {fin. }}$. Hence functions in $\mathcal{M}(\Omega)$ are locally constant. One can show that the dimension of $\mathcal{M}(\Omega)=\left[F_{S}: \Omega\right]$, which is finite.

Let $a \in \mathcal{O}_{S}$ and let $\mathfrak{b} \subset \mathcal{O}_{S}$ be an ideal. Let $\left(\frac{a}{b}\right)$ be the $n$th power residue symbol defined in BBF06. In general this symbol depends on the set of places $S$, but we omit this from the notation. Let $t$ be a positive integer and let $a, c \in \mathcal{O}_{S}$ with $c \neq 0$. Choose a nontrivial additive character $\psi$ of $F_{S}$ such that $\psi\left(x \mathcal{O}_{S}\right)=1$ if and only if $x \in \mathcal{O}_{S}$ [BB06a, Lemma 1]. Then we define a Gauss sum $g\left(a, c ; \epsilon^{t}\right)$ by

$$
g\left(a, c ; \epsilon^{t}\right)=\sum_{d \bmod c} \epsilon^{t}\left(\left(\frac{d}{c \mathcal{O}_{S}}\right)\right) \psi\left(\frac{a d}{c}\right) .
$$

We list some properties of the residue symbol and Gauss sums. For proofs, see [Neu99, Chapter 6/Theorem 8.3] for reciprocity, and Ireland-Rosen [IR90] for the properties of Gauss sums.

Proposition 2.1. Let $a, b, m \in \mathcal{O}_{S}$ with $a, b$ relatively prime. Then

(1) (Reciprocity) $\left(\frac{a}{b}\right)=(b, a)_{S}\left(\frac{b}{a}\right)$.

(2) $g\left(m, a b ; \epsilon^{t}\right)=g\left(m, a ; \epsilon^{t}\right) g\left(m, b ; \epsilon^{t}\right) \epsilon^{t}\left(\left(\frac{a}{b}\right)\left(\frac{b}{a}\right)\right)$.

(3) $g\left(a m, b ; \epsilon^{t}\right)=\epsilon^{-t}\left(\left(\frac{a}{b}\right)\right) g\left(m, b ; \epsilon^{t}\right)$.

(4) If $\varpi \in \mathcal{O}_{S}$ is prime and $t$ does not vanish mod $n$, then

$$
g\left(1, \varpi ; \epsilon^{t}\right) g\left(1, \varpi ; \epsilon^{-t}\right)=|\varpi|
$$

\section{A WeYl GROUP ACTION}

We begin by recalling some basic definitions and properties of root systems. For more details, we refer to Hum90.

Let $\Phi$ be an irreducible reduced root system of rank $r$ with Weyl group $W$. Choose an ordering of the roots and let $\Phi=\Phi^{+} \cup \Phi^{-}$be the decomposition into positive and negative roots. Let $\left\{\alpha_{1}, \alpha_{2}, \ldots, \alpha_{r}\right\}$ be the set of simple roots, and let $\sigma_{i}$ be the Weyl group element corresponding to the reflection through the hyperplane perpendicular to $\alpha_{i}$. Let $\left\{\omega_{1}, \ldots, \omega_{r}\right\}$ be the fundamental weights.

Let $\Lambda$ be the lattice generated by the roots. The Weyl group $W$ acts on $\Lambda$ from the left: $\lambda \mapsto w \lambda$. We choose a $W$-invariant inner product $\langle$,$\rangle on \Lambda \otimes \mathbb{R}$ normalized so that the short roots of $\Phi$ have squared length 1 ; that is, $\langle\alpha, \alpha\rangle=1$ for all short roots $\alpha$. With this convention, the inner product of any two roots is 
always a half-integer 1 Our normalization implies that for $\alpha \in \Phi$, we have

$$
\|\alpha\|^{2}= \begin{cases}1 & \text { for all } \alpha \text { in types } A, D, E \\ 1 & \text { for } \alpha \text { a short root in types } B, C, F_{4}, G_{2}, \\ 2 & \text { for } \alpha \text { a long root in types } B, C, F_{4}, \\ 3 & \text { for } \alpha \text { a long root in type } G_{2} .\end{cases}
$$

Label the nodes of the Dynkin diagram of $\Phi$. We say that nodes $i$ and $j$ are adjacent if $i \neq j$ and $\left(\sigma_{i} \sigma_{j}\right)^{2} \neq 1$. The group $W$ is generated by the simple reflections $\sigma_{i}$, which satisfy the relations $\left(\sigma_{i} \sigma_{j}\right)^{r(i, j)}=1$, where for $1 \leq i, j \leq r$ we have

$$
r(i, j)= \begin{cases}1 & \text { if } i=j \\ 2 & \text { if } i, j \text { are not adjacent, } \\ 3 & \text { if } i, j \text { are adjacent and } \alpha_{i}, \alpha_{j} \text { have the same length, } \\ 4 & \text { if } i, j \text { adjacent, } \Phi \neq G_{2} \text { and } \alpha_{i}, \alpha_{j} \text { have different lengths, } \\ 6 & \text { for } i, j \text { adjacent in type } G_{2} .\end{cases}
$$

Let $c(i, j)=2\left\langle\alpha_{i}, \alpha_{j}\right\rangle /\left\langle\alpha_{j}, \alpha_{j}\right\rangle \in \mathbb{Z}$ be the Cartan integer attached to the simple roots $\alpha_{i}, \alpha_{j}$. These integers encode the action of $W$ on the simple roots:

$$
\sigma_{j}: \alpha_{i} \longmapsto \alpha_{i}-c(i, j) \alpha_{j} .
$$

Let length: $W \rightarrow \mathbb{Z}_{\geq 0}$ be the length function with respect to the generators $\sigma_{1}, \ldots, \sigma_{r}$, and put

$$
\operatorname{sgn}(w)=(-1)^{\operatorname{length}(w)} .
$$

For any $w \in W$, define $\Phi(w)$ by

$$
\Phi(w)=\left\{\alpha \in \Phi^{+} \mid w \alpha \in \Phi^{-}\right\} .
$$

We have length $(w)=|\Phi(w)|$. If $w \in W$ satisfies length $\left(\sigma_{i} w\right)=\operatorname{length}(w)+1$ for a simple reflection $\sigma_{i}$, then

$$
\Phi\left(\sigma_{i} w\right)=\Phi(w) \cup\left\{w^{-1} \alpha_{i}\right\} .
$$

Similarly, if length $\left(w \sigma_{i}\right)=\operatorname{length}(w)+1$ for a simple reflection $\sigma_{i}$, then

$$
\Phi\left(w \sigma_{i}\right)=\sigma_{i}(\Phi(w)) \cup\left\{\alpha_{i}\right\} .
$$

Moreover, define $\rho \in \Lambda \otimes \mathbb{Q}$ by $\rho=\sum \omega_{i}$. Then

$$
\rho-w^{-1} \rho=\sum_{\alpha \in \Phi(w)} \alpha .
$$

Any $\lambda \in \Lambda$ has a unique representation as an integral linear combination of the simple roots

$$
\lambda=k_{1} \alpha_{1}+k_{2} \alpha_{2}+\cdots+k_{r} \alpha_{r}
$$

Let

$$
d(\lambda)=k_{1}+\cdots+k_{r}
$$

be the usual height function on $\Lambda$. Introduce the standard partial ordering on $\Lambda$ by defining $\lambda \succeq 0$ if $\lambda$ is a nonnegative linear combination of the simple roots. Given $\lambda, \lambda^{\prime} \in \Lambda$, define $\lambda \succeq \lambda^{\prime}$ if $\lambda-\lambda^{\prime} \succeq 0$.

\footnotetext{
${ }^{1}$ We remark that this is different from the usual Bourbaki convention, which takes the normalization $\langle\alpha, \alpha\rangle=2$ for all short roots except those in type $B_{r}$, where the short roots satisfy $\langle\alpha, \alpha\rangle=1$.
} 
Our goal in this section is to study a certain Weyl group action. In fact we will define a collection of Weyl group actions indexed by some parameters. The first parameter is a strongly dominant weight $\theta$. The weight $\theta$ determines an $r$-tuple $\ell=\left(l_{1}, \ldots, l_{r}\right)$ of nonnegative integers by $\theta=\sum\left(l_{i}+1\right) \omega_{i}$. We call $\ell$ a twisting parameter; ultimately it will be connected to the global twisting parameter $\mathbf{m}$ in the construction of our multiple Dirichlet series. The weight $\theta$ determines an action of $W$ on the root lattice through affine linear transformations by

$$
w \bullet \lambda=w(\lambda-\theta)+\theta
$$

It is not hard to check that for $w=\sigma_{i}$, a simple reflection, we have

$$
\sigma_{i} \bullet \lambda=\sigma_{i} \lambda+\left(l_{i}+1\right) \alpha_{i} .
$$

The next parameter is a positive integer $n$. The integer $n$ determines a collection of integers $\{m(\alpha)\}_{\alpha \in \Phi^{+}}$by

$$
m(\alpha)=n / \operatorname{gcd}\left(n,\|\alpha\|^{2}\right)
$$

Next we choose a positive integer $q$. Together with $q$ we consider a collection of complex numbers $\gamma(i) \in \mathbb{C}$, indexed by the integers modulo $n$, and such that $\gamma(0)=-1$ and

$$
\gamma(i) \gamma(-i)=1 / q \text { if } i \neq 0 \bmod n \text {. }
$$

Later $q$ will be taken to be the norm of a prime $\varpi$ in $\mathcal{O}_{S}$, and up to a factor of $q$, the number $\gamma(i)$ will be the Gauss sum $g\left(1, \varpi ; \epsilon^{i}\right)$ from (2.1).

We are almost ready to define our action. Choose and fix parameters $(\theta, n, q$, $\{\gamma(i)\})$ as above. Let $A=\mathbb{C}[\Lambda]$ be the ring of Laurent polynomials on the lattice $\Lambda$. Hence $A$ consists of all expressions of the form $f=\sum_{\beta \in \Lambda} c_{\beta} \mathbf{x}^{\beta}$, where $c_{\beta} \in \mathbb{C}$ and almost all are zero, and the multiplication of monomials is defined by addition in $\Lambda: \mathbf{x}^{\beta} \mathbf{x}^{\lambda}=\mathbf{x}^{\beta+\lambda}$. Given $f$, the set of $\left\{\beta \mid c_{\beta} \neq 0\right\}$ is called the support of $f$, and is denoted Supp $f$. We identify $A$ with $\mathbb{C}\left[x_{1}, x_{1}^{-1}, \ldots, x_{r}, x_{r}^{-1}\right]$ via $\mathbf{x}^{\alpha_{i}} \mapsto x_{i}$.

We define a "change of variables" action on $A$ as follows. Write $\mathbf{x}=\left(x_{1}, \ldots, x_{r}\right)$. Then we define $\sigma_{j} \mathbf{x}=\mathbf{x}^{\prime}$, where

$$
\left(\mathbf{x}^{\prime}\right)_{i}=q^{-c(i, j)} x_{i} x_{j}^{-c(i, j)}
$$

Note that $\left(\sigma_{j} \mathbf{x}\right)_{j}=1 /\left(q^{2} x_{j}\right)$ for all $j$, and $\left(\sigma_{j} \mathbf{x}\right)_{i}=x_{i}$ if and only if $\alpha_{i}$ and $\alpha_{j}$ are orthogonal in $\Phi$. It is easy to verify that this action of the simple reflections extends to all of $W$, since it is essentially a reformulation of the standard geometric action of $W$ on $\Lambda \otimes \mathbb{R}$ (cf. (3.2)). One can also easily check that if $f_{\beta}(\mathbf{x})=\mathbf{x}^{\beta}$ is a monomial, then

$$
f_{\beta}(w \mathbf{x})=q^{d\left(w^{-1} \beta-\beta\right)} \mathbf{x}^{w^{-1} \beta} .
$$

Now let $\Lambda^{\prime} \subset \Lambda$ be the sublattice generated by the set $\{m(\alpha) \alpha\}_{\alpha \in \Phi \text {. A direct }}$ computation with Cartan matrices shows that $W$ takes $\Lambda^{\prime}$ into itself. Let $\widetilde{A}$ be the field of fractions of $A$. We have the decomposition

$$
\widetilde{A}=\bigoplus_{\lambda \in \Lambda / \Lambda^{\prime}} \widetilde{A}_{\lambda},
$$

where $\widetilde{A}_{\lambda}$ consists of the functions $f / g(f, g \in A)$ such that Supp $g$ lies in the kernel of the map $\nu: \Lambda \rightarrow \Lambda / \Lambda^{\prime}$, and $\nu$ maps Supp $f$ to $\lambda$. 
We now define the action of $W$ on $\widetilde{A}$ for a generator $\sigma_{i} \in W$. Put $m=m\left(\alpha_{i}\right)$ (cf. (3.7)). For any $\beta \in \Lambda$, define the integer

$$
\mu_{\ell, i}(\beta)=d\left(\sigma_{i} \bullet \beta-\beta\right) \in \mathbb{Z} .
$$

Let $(k)_{m} \in\{0, \ldots, m-1\}$ denote the remainder upon division of $k$ by $m$, and define the rational functions

$$
\begin{aligned}
& \mathcal{P}_{\beta, \ell, i}(x)=(q x)^{l_{i}+1-\left(\mu_{\ell, i}(\beta)\right)_{m}} \frac{1-1 / q}{1-(q x)^{m} / q}, \\
& \mathcal{Q}_{\beta, \ell, i}(x)=-\gamma\left(-\left\|\alpha_{i}\right\|^{2} \mu_{\ell, i}(\beta)\right)(q x)^{l_{i}+1-m} \frac{1-(q x)^{m}}{1-(q x)^{m} / q} .
\end{aligned}
$$

Definition 3.1. Let $f(\mathbf{x}) \in \widetilde{A}_{\beta}$. Define

$$
\left(\left.f\right|_{\ell} \sigma_{i}\right)(\mathbf{x})=\left(\mathcal{P}_{\beta, \ell, i}\left(x_{i}\right)+\mathcal{Q}_{\sigma_{i} \bullet \beta, \ell, i}\left(x_{i}\right)\right) f\left(\sigma_{i} \mathbf{x}\right) \in \widetilde{A} .
$$

We extend this definition linearly to all of $\widetilde{A}$ using (3.10).

Theorem 3.2. The action of the generators (3.14) extends to give an action of $W$ on $\widetilde{A}$. More precisely, the action (3.14) satisfies the defining relations (3.1).

Proof. The proof consists of explicit computations that are very similar to those done in the proof of [CG07, Lemma 3.2]. The main point is that verifying the relations (3.1) amounts to checking certain identities among rational functions. These identities depend only in a minor way on $n$ and are easily implemented on a computer. Since the computations are rather lengthy, we content ourselves with only explicitly presenting some of them here. For the general computations, we merely give an overview and will leave most of the details to the reader.

First we show that the $\sigma_{i}$ act by involutions on $\widetilde{A}$. Let $m=n / \operatorname{gcd}\left(n,\left\|\alpha_{i}\right\|^{2}\right)$ as above. It suffices to check on functions of the form $f(\mathbf{x})=\mathbf{x}^{\beta} / h(\mathbf{x})$, where $h(\mathbf{x}) \in \widetilde{A}_{0}$. We compute (3.14), separate the result into homogeneous terms, and find

$$
\begin{aligned}
\mathcal{P}_{\beta, \ell, i}\left(x_{i}\right) f\left(\sigma_{i} \mathbf{x}\right) & \in \widetilde{A}_{\beta}, \\
\mathcal{Q}_{\sigma_{i} \bullet \beta, \ell, i}\left(x_{i}\right) f\left(\sigma_{i} \mathbf{x}\right) & \in \widetilde{A}_{\sigma_{i} \bullet \beta},
\end{aligned}
$$

where we have abused notation and have denoted the image of $\beta$ in $\Lambda / \Lambda^{\prime}$ by $\beta$ as well. Applying $\sigma_{i}$ again, we find

$$
\begin{aligned}
\left.f\right|_{\ell} \sigma_{i}^{2}=\left(\mathcal{P}_{\beta}(x) \mathcal{P}_{\beta}\left(1 /\left(q^{2} x\right)\right)+\mathcal{Q}_{\sigma_{i} \bullet \beta}(x) \mathcal{P}_{\beta}\left(1 /\left(q^{2} x\right)\right)\right. \\
\left.+\mathcal{P}_{\sigma_{i} \bullet \beta}(x) \mathcal{Q}_{\sigma_{i} \bullet \beta}\left(1 /\left(q^{2} x\right)\right)+\mathcal{Q}_{\beta}(x) \mathcal{Q}_{\sigma_{i} \bullet \beta}\left(1 /\left(q^{2} x\right)\right)\right) f .
\end{aligned}
$$

In (3.17) we lightened the notation by removing $\ell, i$ from $\mathcal{P}, \mathcal{Q}$ and by writing $x$ for $x_{i}$. Now using the identities

$$
\mu_{\ell, i}(\beta)=-\mu_{\ell, i}\left(\sigma_{i} \bullet \beta\right)
$$

and

$$
(-k)_{m}= \begin{cases}0 & \text { if } m \mid k \\ m-k & \text { otherwise }\end{cases}
$$


we can check that the sum of the four products of $\mathcal{P}, \mathcal{Q}$ on the right of (3.17) equals 1. Indeed, there are two cases to consider, according to whether $\mu_{\ell, i}(\beta)$ vanishes $\bmod m$ or not. In the second case we have

$$
\begin{aligned}
\mathcal{Q}_{\sigma_{i} \bullet \beta}(x) \mathcal{P}_{\beta}\left(1 /\left(q^{2} x\right)\right)+\mathcal{P}_{\sigma_{i} \bullet \beta}(x) \mathcal{Q}_{\sigma_{i} \bullet \beta}\left(1 /\left(q^{2} x\right)\right) & =0, \\
\mathcal{P}_{\beta}(x) \mathcal{P}_{\beta}\left(1 /\left(q^{2} x\right)\right)+\mathcal{Q}_{\beta}(x) \mathcal{Q}_{\sigma_{i} \bullet \beta}\left(1 /\left(q^{2} x\right)\right) & =1,
\end{aligned}
$$

and in the first case there are nontrivial cancellations among all four terms that lead to the desired sum. This shows that the $\sigma_{i}$ act by involutions.

Now we explain how to prove that the rest of (3.1) holds. We consider a relation of the form $\sigma_{i} \sigma_{j} \sigma_{i}=\sigma_{j} \sigma_{i} \sigma_{j}$. This is only possible if $\left\|\alpha_{i}\right\|=\left\|\alpha_{j}\right\|$, and so without loss of generality we can assume $m=n$.

We again put $f(\mathbf{x})=\mathbf{x}^{\beta} / h(\mathbf{x})$ and compute $f_{1}(\mathbf{x}):=\left(\left.f\right|_{\ell} \sigma_{i} \sigma_{j} \sigma_{i}\right)(\mathbf{x})$ and $f_{2}(\mathbf{x}):=$ $\left(\left.f\right|_{\ell} \sigma_{j} \sigma_{i} \sigma_{j}\right)(\mathbf{x})$. Each of these expands to a sum of eight products. Each product consists of three factors, and the factors are $\mathcal{P}$ 's and $\mathcal{Q}$ 's with various inputs. In both $f_{1}$ and $f_{2}$ we eliminate a common denominator coming from $h(\mathbf{x})$, and then we can factor out $\mathbf{x}^{\sigma_{i} \sigma_{j} \sigma_{i} \beta}$, some common $q$-powers, and some common monomials coming from the twisting parameter. After this we find that the eight remaining terms in $f_{1}$ are

$$
\begin{array}{r}
\mathcal{P}_{\sigma_{j} \bullet \beta, i}\left(x_{i}\right) \mathcal{Q}_{\sigma_{j} \bullet \beta, j}\left(q x_{i} x_{j}\right) \mathcal{P}_{\beta, i}\left(x_{j}\right), \quad \mathcal{P}_{\sigma_{j} \sigma_{i} \bullet \beta, i}\left(x_{i}\right) \mathcal{Q}_{\sigma_{j} \sigma_{i} \bullet \beta, j}\left(q x_{i} x_{j}\right) \mathcal{Q}_{\sigma_{i} \bullet \beta, i}\left(x_{j}\right), \\
\mathcal{Q}_{\sigma_{i} \bullet \beta, i}\left(x_{i}\right) \mathcal{P}_{\beta, j}\left(q x_{i} x_{j}\right) \mathcal{P}_{\beta, i}\left(x_{j}\right), \quad \mathcal{Q}_{\beta, i}\left(x_{i}\right) \mathcal{P}_{\sigma_{i} \bullet \beta, j}\left(q x_{i} x_{j}\right) \mathcal{Q}_{\sigma_{i} \bullet \beta, i}\left(x_{j}\right), \\
\mathcal{Q}_{\sigma_{i} \sigma_{j} \bullet \beta, i}\left(x_{i}\right) \mathcal{Q}_{\sigma_{j} \bullet \beta, j}\left(q x_{i} x_{j}\right) \mathcal{P}_{\beta, i}\left(x_{j}\right), \\
\mathcal{Q}_{\sigma_{i} \sigma_{j} \sigma_{i} \bullet \beta, i}\left(x_{i}\right) \mathcal{Q}_{\sigma_{j} \sigma_{i} \sigma_{i} \bullet \beta, j}\left(q x_{i} x_{j}\right) \mathcal{Q}_{\sigma_{i} \bullet \beta, i}\left(x_{j}\right),
\end{array}
$$

and that $f_{2}$ consists of the same eight terms with $i$ and $j$ switched. Here we again eliminate $\ell$ from the notation. Note that each term in $f_{1}, f_{2}$ can be uniquely identified by giving a length-three word in $\mathcal{P}$ and $\mathcal{Q}$ and saying whether it appears in $f_{1}$ or $f_{2}$. The first term in (3.18), for example, can be encoded as $(\mathcal{P} \mathcal{P} \mathcal{P})_{1}$.

In summary, to prove $f_{1}=f_{2}$, we need to verify the identity of rational functions

$$
(\mathcal{P} \mathcal{P} \mathcal{P})_{1}+\cdots+(\mathcal{Q Q} \mathcal{Q})_{1}=(\mathcal{P P} \mathcal{P})_{2}+\cdots+(\mathcal{Q Q} \mathcal{Q})_{2}
$$

This can be done as follows. Let $\mu_{i}=\mu_{\ell, i}(\beta)$ and $\mu_{j}=\mu_{\ell, j}(\beta)$. Then the identities needed to check (3.19) depend in a minor way on $\mu_{i}, \mu_{j} \bmod n$ :

(1) We have $(\mathcal{P} \mathcal{P} \mathcal{P})_{1}=(\mathcal{P} \mathcal{P} \mathcal{P})_{2},(\mathcal{Q Q} \mathcal{Q})_{1}=(\mathcal{Q Q} \mathcal{Q})_{2},(\mathcal{P} \mathcal{Q} \mathcal{Q})_{1}=(\mathcal{Q} \mathcal{Q P})_{2}$, and $(\mathcal{Q Q P})_{1}=(\mathcal{P} \mathcal{Q} \mathcal{Q})_{2}$, independent of $\mu_{i}, \mu_{j} \bmod n$.

(2) If $\mu_{i}$ and $\mu_{j}$ are nonzero $\bmod n$, then $(\mathcal{Q P} \mathcal{Q})_{1}=(\mathcal{Q P} \mathcal{Q})_{2},(\mathcal{P P} \mathcal{Q})_{1}+$ $(\mathcal{Q P} \mathcal{P})_{1}=(\mathcal{P} \mathcal{Q P})_{2}$, and $(\mathcal{P Q P})_{1}=(\mathcal{P P} \mathcal{Q})_{2}+(\mathcal{Q P} \mathcal{P})_{2}$

(3) If $\mu_{i}=0 \bmod n$ and $\mu_{j} \neq 0 \bmod n$, then $(\mathcal{P} \mathcal{Q P})_{1}=(\mathcal{P} \mathcal{P} \mathcal{Q})_{2}+(\mathcal{Q P} \mathcal{P})_{2}$ and $(\mathcal{P P} \mathcal{Q})_{1}+(\mathcal{Q P P})_{1}+(\mathcal{Q P} \mathcal{Q})_{1}=(\mathcal{P} \mathcal{Q P})_{2}+(\mathcal{Q P} \mathcal{Q})_{2}$.

(4) If $\mu_{i} \neq 0 \bmod n$ and $\mu_{j}=0 \bmod n$, then we have the same identities as in case 3. but with 1 and 2 switched.

These identities prove (3.19), which implies $\left(\left.f\right|_{\ell} \sigma_{i} \sigma_{j} \sigma_{i}\right)(\mathbf{x})=\left(\left.f\right|_{\ell} \sigma_{j} \sigma_{i} \sigma_{j}\right)(\mathbf{x})$.

Lengthier but entirely similar computations show that the relations $\left(\sigma_{i} \sigma_{j}\right)^{4}=1$ and $\left(\sigma_{1} \sigma_{2}\right)^{6}=1$ (for $\Phi=G_{2}$ ) hold, as long as $n$ is relatively prime to all squared root lengths. It is also easy to check the required relations of the form $\left(\sigma_{i} \sigma_{j}\right)^{2}=1$ if $\alpha_{i}$ and $\alpha_{j}$ have the same length. 
If $n$ is not relatively prime to all squared root lengths, then the computations are only slightly more complicated. For instance, assume that $n=2 m$ is even and that $\sigma_{i}, \sigma_{j}$ satisfy $\left(\sigma_{i} \sigma_{j}\right)^{4}=1$. Assume $\left\|\alpha_{i}\right\|^{2}=2,\left\|\alpha_{j}\right\|^{2}=1$, and let $f=\mathbf{x}^{\beta} / h(\mathbf{x})$ as before. Apply $\sigma_{i}$ to $f$ as in (3.15). One can then use the distribution relation

$$
\frac{1}{1-z}=\frac{1}{1-z^{2}}+\frac{z}{1-z^{2}}
$$

to write each of the $\mathcal{P}$ and $\mathcal{Q}$ terms on the right of (3.15) (which have denominator $\left.1-(q x)^{m} / q\right)$ as sums of two terms with denominator $1-(q x)^{2 m} / q^{2}$. The resulting two terms from $\mathcal{P}$ lie in $\widetilde{A}_{\beta}$ and $\widetilde{A}_{\beta+m \alpha_{i}}$, whereas the two terms from $\mathcal{Q}$ lie in $\widetilde{A}_{\sigma_{i} \bullet \beta}$ and $\widetilde{A}_{\sigma_{i} \bullet \beta+m \alpha_{i}}$ (here the congruence classes should be taken in $\Lambda / n \Lambda$, not $\Lambda / \Lambda^{\prime}$ ). Hence the computation is essentially the same as that for $n$ odd, except that each application of $\sigma_{i}$ to $f$ results in a sum of four terms instead of the two in (3.15). The computation for $G_{2}$ is similar, except that one replaces 3.20) with an identity with denominator $1-z^{3}$. Finally, the same trick works to check the required relations of the form $\left(\sigma_{i} \sigma_{j}\right)^{2}=1$ when $\alpha_{i}$ and $\alpha_{j}$ have different lengths.

We are now about to prove the main theorem of this section, but before doing so we require some notation. Define

$$
\Delta(\mathbf{x})=\prod_{\alpha>0}\left(1-q^{m(\alpha) d(\alpha)} \mathbf{x}^{m(\alpha) \alpha}\right) \quad \text { and } \quad D(\mathbf{x})=\prod_{\alpha>0}\left(1-q^{m(\alpha) d(\alpha)-1} \mathbf{x}^{m(\alpha) \alpha}\right) .
$$

Put

$$
j(w, \mathbf{x})=\Delta(\mathbf{x}) / \Delta(w \mathbf{x}) .
$$

This function satisfies the 1-cocycle relation

$$
j\left(w w^{\prime}, \mathbf{x}\right)=j\left(w, w^{\prime} \mathbf{x}\right) j\left(w^{\prime}, \mathbf{x}\right)
$$

and can be explicitly computed as follows:

Lemma 3.3. We have

$$
j(w, \mathbf{x})=\operatorname{sgn}(w) q^{d(\beta)} \mathbf{x}^{\beta},
$$

where

$$
\beta=\sum_{\alpha \in \Phi(w)} m(\alpha) \alpha
$$

Proof. Using (3.9) we have

$$
\begin{aligned}
\Delta(w \mathbf{x})= & \prod_{\alpha>0}\left(1-q^{m\left(w^{-1} \alpha\right) d\left(w^{-1} \alpha\right)} \mathbf{x}^{m\left(w^{-1} \alpha\right) w^{-1} \alpha}\right) \\
= & \prod_{\substack{\alpha>0 \\
w^{-1} \alpha<0}}\left(1-q^{m\left(w^{-1} \alpha\right) d\left(w^{-1} \alpha\right)} \mathbf{x}^{m\left(w^{-1} \alpha\right) w^{-1} \alpha}\right) \\
& \times \prod_{\substack{\alpha>0 \\
w^{-1} \alpha>0}}\left(1-q^{m\left(w^{-1} \alpha\right) d\left(w^{-1} \alpha\right)} \mathbf{x}^{m\left(w^{-1} \alpha\right) w^{-1} \alpha}\right) \\
= & \prod_{\substack{\alpha<0 \\
w \alpha>0}}\left(1-q^{m(\alpha) d(\alpha)} \mathbf{x}^{m(\alpha) \alpha}\right) \prod_{\substack{\alpha>0 \\
w \alpha>0}}\left(1-q^{m(\alpha) d(\alpha)} \mathbf{x}^{m(\alpha) \alpha}\right) \\
= & \prod_{\substack{\alpha>0 \\
w \alpha<0}}\left(1-q^{-m(\alpha) d(\alpha)} \mathbf{x}^{-m(\alpha) \alpha}\right) \prod_{\substack{\alpha>0 \\
w \alpha>0}}\left(1-q^{m(\alpha) d(\alpha)} \mathbf{x}^{m(\alpha) \alpha}\right),
\end{aligned}
$$


where we obtain the third equality above with the substitution $\alpha \mapsto w^{-1} \alpha$ in both products. Thus $j(w, \mathbf{x})$ is equal to the quotient

$$
\Delta(\mathbf{x}) /\left(\prod_{\alpha \in \Phi(w)}\left(1-q^{-m(\alpha) d(\alpha)} \mathbf{x}^{-m(\alpha) \alpha}\right) \cdot \prod_{\substack{\alpha>0 \\ \alpha \notin \Phi(w)}}\left(1-q^{m(\alpha) d(\alpha)} \mathbf{x}^{m(\alpha) \alpha}\right)\right) .
$$

Multiplying the top and bottom of the last expression by

$$
\prod_{\alpha \in \Phi(w)}-q^{m(\alpha) d(\alpha)} \mathbf{x}^{m(\alpha) \alpha}=(-1)^{|\Phi(w)|} \prod_{\alpha \in \Phi(w)} q^{m(\alpha) d(\alpha)} \mathbf{x}^{m(\alpha) \alpha}
$$

converts the denominator to $\Delta(\mathbf{x})$. After dividing we find

$$
j(w, \mathbf{x})=(-1)^{|\Phi(w)|} \prod_{\alpha \in \Phi(w)} q^{m(\alpha) d(\alpha)} \mathbf{x}^{m(\alpha) \alpha} .
$$

This agrees with the statement, since $|\Phi(w)|=\operatorname{length}(w)$.

We list some further properties of the group action that we will use.

Lemma 3.4. Let $\ell$ be an $r$-tuple of nonnegative integers.

(1) Let $f \in \widetilde{A}$. Let $g(\mathbf{x}) \in \widetilde{A}_{\beta}$ with $\sigma_{i} \beta-\beta=0 \in \Lambda / \Lambda^{\prime}$. Then

Similarly, if $\beta \in \Lambda^{\prime}$, then for all $w \in W$,

$$
\left(\left.g f\right|_{\ell} w\right)(\mathbf{x})=g(w \mathbf{x})\left(\left.f\right|_{\ell} w\right)(\mathbf{x}) .
$$

(2) The function $j(w, \mathbf{x})\left(\left.1\right|_{\ell} w\right)(\mathbf{x})$ is regular at the origin.

(3) Suppose length $\left(\sigma_{i} w\right)=\operatorname{length}(w)+1$. Then

$$
j\left(\sigma_{i} w, \mathbf{x}\right)\left(\left.\mathbf{x}^{\left(l_{i}+1-m\left(\alpha_{i}\right)\right) \alpha_{i}}\right|_{\ell} w\right)(\mathbf{x})
$$

is regular at the origin.

Proof. Since $W$ preserves the sublattice $\Lambda^{\prime}$, equation (3.22) is an easy consequence of (3.21), so we prove (3.21). It suffices to prove (3.21) for $f(\mathbf{x}) \in \widetilde{A}_{\lambda}$. Then $g f$ is still in $\widetilde{A}_{\lambda}$, so (3.21) follows easily from Definition (3.14) of the $\left.\right|_{\ell}$ action. (The

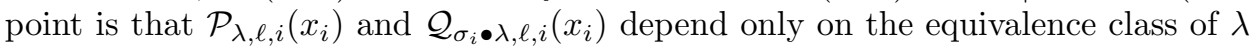
in $\Lambda / \Lambda^{\prime}$.)

Statements (2) and (3) are proved simultaneously by induction. We will show the details for the proof of (2); the proof of (3) is similar.

First of all, both statements are obviously true when length $(w)=0$. Now assume $\sigma_{i} w$ satisfies length $\left(\sigma_{i} w\right)=$ length $(w)+1$, let $m=m\left(\alpha_{i}\right)$, and assume that (2), (3) are true for $w$. We prove that $j\left(\sigma_{i} w, \mathbf{x}\right)\left(\left.1\right|_{\ell} \sigma_{i} w\right)(\mathbf{x})$ is regular. Indeed, we have

$$
j\left(\sigma_{i} w, \mathbf{x}\right)\left(\left.1\right|_{\ell} \sigma_{i} w\right)(\mathbf{x})=j\left(\sigma_{i} w, \mathbf{x}\right)\left(\left.\left[\mathcal{P}_{0, \ell, i}\left(x_{i}\right)+\mathcal{Q}_{\sigma \bullet 0, \ell, i}\left(x_{i}\right)\right]\right|_{\ell} w\right)(\mathbf{x}),
$$

and it suffices to check the regularity of the $\mathcal{P}$ and $\mathcal{Q}$ terms separately in (3.23).

Now $\mathcal{P}_{0, \ell, i}\left(x_{i}\right) \in \widetilde{A}_{0}$, so we can apply (3.22) with $f=1, g=\mathcal{P}_{0, \ell, i}\left(x_{i}\right)$. Thus, abbreviating $P(\mathbf{x})=\mathcal{P}_{0, \ell, i}\left(x_{i}\right)$, we have

$$
\left(\left.P \cdot 1\right|_{\ell} w\right)(\mathbf{x})=P(w \mathbf{x})\left(\left.1\right|_{\ell} w\right)(\mathbf{x}) .
$$

Up to irrelevant $q$-powers, this expression can be written as

$$
\frac{\mathbf{x}^{k \delta}}{1-q^{m-1} \mathbf{x}^{\delta}}\left(\left.1\right|_{\ell} w\right)(\mathbf{x})
$$


where $\delta=m w^{-1}\left(\alpha_{i}\right) \in \Lambda$ and $k=\left(l_{i}+1-\left(l_{i}+1\right)_{m}\right) / m$ is nonnegative. By the description of $\Phi\left(\sigma_{i} w\right)$ in (3.3), we have $w^{-1}\left(\alpha_{i}\right)>0$. Therefore the rational function in (3.24) is regular at the origin. By the induction hypothesis, $j(w, \mathbf{x})\left(\left.1\right|_{\ell} w\right)(\mathbf{x})$ has no pole at the origin, and so we only need to check that $j\left(\sigma_{i} w, \mathbf{x}\right)\left(\left.1\right|_{\ell} w\right)(\mathbf{x})$ has no pole at the origin. Looking again at (3.3), we find

$$
\sum_{\alpha \in \Phi\left(\sigma_{i} w\right)} m(\alpha) \alpha \succ \sum_{\alpha \in \Phi(w)} m(\alpha) \alpha
$$

Hence by Lemma 3.3 the ratio $j\left(\sigma_{i} w, \mathbf{x}\right) / j(w, \mathbf{x})$ is a monomial of positive degree. Therefore $j\left(\sigma_{i} w, \mathbf{x}\right)\left(\left.1\right|_{\ell} w\right)(\mathbf{x})$ has no pole at the origin. This completes the analysis of the $\mathcal{P}$ term.

We can treat the $\mathcal{Q}$ term in (3.23) by a similar argument. We can apply (3.22) by taking $f=x_{i}^{l_{i}+1-m}, g=\mathcal{Q}_{\sigma_{i} \bullet 0, \ell, i}\left(x_{i}\right) / f$. Applying $\left.\right|_{\ell} w$ to $g f$, we obtain a rational function with no pole at the origin times

$$
\left(\left.x_{i}^{l_{i}+1-m}\right|_{\ell} w\right)(\mathbf{x}) .
$$

By induction, this becomes regular at the origin after multiplying by $j\left(\sigma_{i} w, \mathbf{x}\right)$. Therefore this case of (2) follows from prior cases of (2), (3). Similar computations work to show that (3) follows from prior cases of (2), (3).

Now define the rational function

$$
h(\mathbf{x} ; \ell)=\Delta(\mathbf{x})^{-1} \sum_{w \in W} j(w, \mathbf{x})\left(\left.1\right|_{\ell} w\right)(\mathbf{x}) \in \widetilde{A} .
$$

The main result of this section is the following theorem:

Theorem 3.5. Let $\ell=\left(l_{1}, \ldots, l_{r}\right)$ with each $l_{i} \geq 0$. The rational function $h=$ $h(\mathbf{x} ; \ell)$ satisfies $\left.h\right|_{\ell} w=h$ for all $w \in W$. Furthermore $N(\mathbf{x} ; \ell)=h(\mathbf{x} ; \ell) D(\mathbf{x})$ is a polynomial in the $x_{i}$ 's. Finally, if $\ell=(0, \ldots, 0)$ and $m=m\left(\alpha_{i}\right)$, then

$$
h\left(0, \ldots, 0, x_{i}, 0, \ldots, 0 ; \ell\right)=\frac{1+\gamma\left(\left\|\alpha_{i}\right\|^{2}\right) q x_{i}}{1-q^{m-1} x_{i}^{m}} .
$$

Proof. The invariance of $h$ is easy to see. Indeed, we have

$$
h(\mathbf{x} ; \ell)=\sum_{w \in W} \frac{1}{\Delta(w \mathbf{x})}\left(\left.1\right|_{\ell} w\right)(\mathbf{x}) .
$$

For any $u \in W$, after applying $\left.\right|_{\ell} u$ and using Lemma 3.4 (11), we see that

$$
\left(\left.h\right|_{\ell} u\right)(\mathbf{x})=\sum_{w \in W} \frac{1}{\Delta(w u \mathbf{x})}\left(\left.1\right|_{\ell} w u\right)(\mathbf{x}) .
$$

Thus $h$ is invariant under the $\left.\right|_{\ell \text {-action. }}$

To prove that $N(\mathbf{x} ; \ell)$ is a polynomial, introduce for each $w \in W$ the rational function

$$
P_{w}(\mathbf{x})=j(w, \mathbf{x})\left(\left.1\right|_{\ell} w\right) D_{w}(\mathbf{x})
$$

where

$$
D_{w}(\mathbf{x})=\prod_{\alpha \in \Phi(w)}\left(1-q^{m(\alpha) d(\alpha)-1} \mathbf{x}^{m(\alpha) \alpha}\right) .
$$

We will show by induction on the length of $w$ that each $P_{w}(\mathbf{x})$ is a polynomial. If $w$ is the identity there is nothing to prove. 
Suppose that for $w \in W$, the rational function $P_{w}(\mathbf{x})$ is a polynomial. Let $\sigma_{i}$ be a simple reflection such that length $\left(w \sigma_{i}\right)=\operatorname{length}(w)+1$. Then

$$
\begin{aligned}
P_{w \sigma_{i}}(\mathbf{x}) & =j\left(w \sigma_{i}, \mathbf{x}\right)\left(\left.1\right|_{\ell} w \sigma_{i}\right)(\mathbf{x}) D_{w \sigma_{i}}(\mathbf{x}) \\
& =j\left(\sigma_{i}, \mathbf{x}\right)\left(\left.\frac{P_{w}}{D_{w}}\right|_{\ell} \sigma_{i}\right)(\mathbf{x}) \cdot D_{w \sigma_{i}}(\mathbf{x}) \\
& =\frac{j\left(\sigma_{i}, \mathbf{x}\right)\left(\left.P_{w}\right|_{\ell} \sigma_{i}\right)(\mathbf{x})}{D_{w}\left(\sigma_{i} \mathbf{x}\right)} \cdot D_{w \sigma_{i}}(\mathbf{x}), \text { by Lemma 3.4 (1) } .
\end{aligned}
$$

By the definition (3.14) of the action of $\sigma_{i}$, we can write $\left(\left.P_{w}\right|_{\ell} \sigma_{i}\right)(\mathbf{x})$ as $P_{w}^{\prime}(\mathbf{x}) /(1-$ $\left.q^{m\left(\alpha_{i}\right)-1} x_{i}^{m\left(\alpha_{i}\right)}\right)$, where $P_{w}^{\prime}$ is a Laurent polynomial in the $x_{i}$. However, as Lemma 3.4 (2) implies that $P_{w}(\mathbf{x})$ is regular at the origin, it follows that $P_{w}^{\prime}$ is a polynomial. Moreover, the denominator $D_{w}\left(\sigma_{i} \mathbf{x}\right)$ is equal to

$$
\prod_{\alpha \in \Phi(w)}\left(1-q^{m(\alpha) d\left(\sigma_{i} \alpha\right)-1} \mathbf{x}^{m(\alpha) \sigma_{i} \alpha}\right)=D_{w \sigma_{i}}(\mathbf{x}) /\left(1-q^{m\left(\alpha_{i}\right)-1} x_{i}^{m\left(\alpha_{i}\right)}\right),
$$

where here we use (3.4) to compute $D_{w \sigma_{i}}(\mathbf{x})$. Note also that $m(\alpha)=m(w \alpha)$ for all $w \in W, \alpha \in \Phi$, since the Weyl group preserves root lengths. Plugging (3.28) back into (3.27), we conclude that

$$
j\left(w \sigma_{i}, \mathbf{x}\right)\left(\left.1\right|_{\ell} w \sigma_{i}\right)(\mathbf{x}) D_{w \sigma_{i}}(\mathbf{x})=j\left(\sigma_{i}, \mathbf{x}\right) P_{w}^{\prime}(\mathbf{x})
$$

is a polynomial. Therefore $h(\mathbf{x} ; \ell) D(\mathbf{x}) \Delta(\mathbf{x})=N(\mathbf{x} ; \ell) \Delta(\mathbf{x})$ is a polynomial.

To complete the proof that $N(\mathbf{x} ; \ell)$ is a polynomial, we check that

$$
N(\mathbf{x} ; \ell) \Delta(\mathbf{x})=D(\mathbf{x}) \sum_{w \in W} j(w, \mathbf{x})\left(\left.1\right|_{\ell} w\right)(\mathbf{x})
$$

is divisible by $\Delta(\mathbf{x})$. We begin with the following simple computation. Let $B_{\lambda}(\mathbf{x})=$ $\mathbf{x}^{\lambda}$ be a monomial with nonnegative exponents. Let $\sigma_{i}$ be a simple reflection and let $m=m\left(\alpha_{i}\right)$. Assume $\sigma_{i} \lambda \succ \lambda$. Then we claim that

$$
B_{\lambda}(\mathbf{x})+j\left(\sigma_{i}, \mathbf{x}\right)\left(\left.B_{\lambda}\right|_{\ell} \sigma_{i}\right)(\mathbf{x})
$$

can be written as a rational function with numerator divisible by $1-q^{m} x_{i}^{m}$ and with denominator $1-q^{m-1} x_{i}^{m}$. Indeed, we have that (3.30) equals

$$
\begin{aligned}
\mathbf{x}^{\lambda}-\left(q x_{i}\right)^{m} q^{d\left(\sigma_{i} \lambda-\lambda\right)} \mathbf{x}^{\sigma_{i} \lambda}\left(\mathcal{P}_{\lambda, \ell, i}\left(x_{i}\right)+\mathcal{Q}_{\sigma_{i} \bullet \lambda, \ell, i}\left(x_{i}\right)\right) \\
\quad=\mathbf{x}^{\lambda}\left(\left(1-q^{m-1} x_{i}^{m}\right)-q^{d\left(\sigma_{i} \lambda-\lambda\right)} \mathbf{x}^{\sigma_{i} \lambda-\lambda}(X+Y)\right),
\end{aligned}
$$

where

$$
\begin{aligned}
X & =\left(q x_{i}\right)^{l_{i}+1+m-(\mu)_{m}}(1-1 / q) /\left(1-q^{m-1} x_{i}^{m}\right), \\
Y & =\gamma\left(q x_{i}\right)^{l_{i}+1}\left(1-q^{m} x_{i}^{m}\right) /\left(1-q^{m-1} x_{i}^{m}\right), \quad \gamma \in \mathbb{C}, \\
\mu & =\mu_{\ell, i}\left(\sigma_{i} \bullet \lambda\right) .
\end{aligned}
$$

It is clear that the $Y$ term has the correct denominator and is divisible by $1-q^{m} x_{i}^{m}$. For the $X$ term, after bringing it together with the initial term we find an expression of the form

$$
\left(1-q^{m-1} x_{i}^{m}+q^{k m-1} x_{i}^{k m}-\left(q x_{i}\right)^{k m}\right) /\left(1-q^{m-1} x_{i}^{m}\right), \quad k \geq 1,
$$

which is a rational function with the required denominator and with numerator divisible by $1-q^{m} x_{i}^{m}$. 
We claim this computation shows that $N(\mathbf{x} ; \ell) \Delta(\mathbf{x})$ is divisible by $1-q^{m} x_{i}^{m}$. Indeed, to see this we use the cocycle property of $j$ to write (3.29) as a double sum, with the inner sum over a set of minimal length representatives for the right cosets of $\sigma_{i}$ in $W$, and with the outer sum running over $1, \sigma_{i}$. After multiplying by $D(\mathbf{x})$, the inner sum becomes a polynomial, and then the computation above shows that after applying the operator $1+j\left(\sigma_{i}, \mathbf{x}\right)\left(\left.\right|_{\ell} \sigma_{i}\right)$ we obtain a rational function with denominator killed by $D(\mathbf{x})$ and with numerator divisible by $1-q^{m} x_{i}^{m}$. This also implies that (3.29) is divisible by $1-q^{m(\alpha)} \mathbf{x}^{m(\alpha) \alpha}$ for any simple root $\alpha$.

We can now show that $1-q^{d(\alpha) m(\alpha)} \mathbf{x}^{m(\alpha) \alpha}$ divides $h(\mathbf{x} ; \ell) \Delta(\mathbf{x})$ for all positive roots $\alpha$. Indeed, write

$$
h(\mathbf{x} ; \ell) \Delta(\mathbf{x})=\left(1-q^{m\left(\alpha_{i}\right)} x_{i}^{m\left(\alpha_{i}\right)}\right) h_{0}(\mathbf{x}),
$$

say, where $D(\mathbf{x}) h_{0}(\mathbf{x})$ is a polynomial and the simple root $\alpha_{i}$ has the same length as $\alpha$. Let $w \in W$ map $\alpha_{i}$ to $\alpha$. Act on both sides of (3.31) by $w$. Then $\pm h(\mathbf{x} ; \ell) \Delta(\mathbf{x})=$ $\left(1-q^{d(\alpha) m(\alpha)} \mathbf{x}^{m(\alpha) \alpha}\right)\left(h_{0} \mid w\right)$, by Lemma 3.4 (1). But, arguing inductively as in the first part of the proof of the theorem, $D(\mathbf{x}) \cdot\left(h_{0} \mid w\right)$ is still polynomial. We conclude that $h(\mathbf{x} ; \ell) D(\mathbf{x}) \Delta(\mathbf{x})$ is divisible by $1-q^{d(\alpha) m(\alpha)} \mathbf{x}^{m(\alpha) \alpha}$ for every positive root $\alpha$. This completes the proof of the polynomiality of $N(\mathbf{x} ; \ell)$.

Now we prove (3.26). Let $\ell=(0, \ldots, 0)$ and set all variables of $h$ equal to zero except for $x_{i}$ in (3.25). The sum over $W$ reduces to two terms, namely $w \in\left\{1, \sigma_{i}\right\}$, since all other terms are easily seen to vanish by Lemma 3.3. Let $m=m\left(\alpha_{i}\right)$. Then $\Delta(\mathbf{x})$ becomes $1-\left(q x_{i}\right)^{m}$, since this is the only factor of $\Delta$ involving a monomial in $x_{i}$ alone. Combining (3.14) and (3.25) we find

$$
\Delta(\mathbf{x})^{-1}\left(1+j\left(\sigma_{i}, \mathbf{x}\right)\left(\left.1\right|_{\ell} \sigma_{i}\right)(\mathbf{x})\right)=\left(1-\left(q x_{i}\right)^{m}\right)^{-1}\left(\mathcal{P}_{0, \ell, i}+\mathcal{Q}_{\sigma_{i} \bullet 0, \ell, i}\right) .
$$

We have $j\left(\sigma_{i}, \mathbf{x}\right)=-\left(q x_{i}\right)^{m}$ and $\mu_{\ell, i}(0)=-\mu_{\ell, i}\left(\alpha_{i}\right)=1$. Thus the right of (3.32) becomes

$$
\frac{1}{1-\left(q x_{i}\right)^{m}}\left(1+\frac{-\left(q x_{i}\right)^{m}(1-1 / q)+\left(q x_{i}\right)\left(1-\left(q x_{i}\right)^{m}\right) \gamma\left(\left\|\alpha_{i}\right\|^{2}\right)}{1-\left(q x_{i}\right)^{m} / q}\right),
$$

which after a short computation is easily seen to be the right of (3.26). This completes the proof of the theorem.

Write $N(\mathbf{x} ; \ell)=\sum_{\lambda \in \Lambda} a_{\lambda} \mathbf{x}^{\lambda}$. Given any $\beta \in \Lambda$ and a simple root $\alpha_{i}$, we define

$$
S_{\beta}=S_{\beta, i}=\left\{\beta+k m \alpha_{i} \mid k \in \mathbb{Z}\right\},
$$

where $m=m\left(\alpha_{i}\right)$. Define

$$
N_{\beta, i}(\mathbf{x})=\sum_{\lambda \in S_{\beta}} a_{\lambda} \mathbf{x}^{\lambda}
$$

Now choose $\beta \in \Lambda$ and assume $\sigma_{i} \bullet \beta=\beta+k \alpha_{i}$ with $k \geq 0$. Define $\delta=(k)_{m}$. Define

$$
f_{\beta, i}(\mathbf{x})= \begin{cases}\left(N_{\beta, i}(\mathbf{x})-\gamma(-\delta)\left(q x_{i}\right)^{m-\delta} N_{\sigma_{i} \bullet \beta, i}(\mathbf{x})\right) /\left(1-q^{m-1} x_{i}^{m}\right) & \text { if } \delta \neq 0 \\ N_{\beta, i}(\mathbf{x}) /\left(1-q^{m-1} x_{i}^{m}\right) & \text { otherwise. }\end{cases}
$$

The function $f_{\beta, i}(\mathbf{x})$ satisfies the following symmetry with respect to the reflection $\sigma_{i}$ : 
Theorem 3.6. We have

$$
\frac{f_{\beta, i}(\mathbf{x})}{f_{\beta, i}\left(\sigma_{i} \mathbf{x}\right)}= \begin{cases}\left(q x_{i}\right)^{l_{i}+1-\delta} & \text { if } \delta \neq 0, \\ \left(q x_{i}\right)^{l_{i}+1-m} & \text { otherwise. }\end{cases}
$$

Proof. We prove the statement when $\delta \neq 0$; the remaining case is simpler and requires no new ideas.

We begin by defining

$$
\begin{aligned}
F_{\beta, i}(\mathbf{x}) & =\left(N_{\beta, i}(\mathbf{x})+N_{\sigma_{i} \bullet \beta, i}(\mathbf{x})\right) /\left(1-q^{m-1} x_{i}^{m}\right) \\
& =\left(\sum_{\lambda \in S_{\beta}} a_{\lambda} \mathbf{x}^{\lambda}+\sum_{\mu \in S_{\sigma_{i}} \bullet \beta} a_{\mu} \mathbf{x}^{\mu}\right) /\left(1-q^{m-1} x_{i}^{m}\right) .
\end{aligned}
$$

By the construction of $h$ and Lemma 3.4 $F_{\beta, i}(\mathbf{x})$ is invariant under $\left.\right|_{\ell} \sigma_{i}$. On the other hand, explicitly applying $\sigma_{i}$ to $F$ yields

$$
\begin{aligned}
\left(\left.F_{\beta, i}\right|_{\ell} \sigma_{i}\right)(\mathbf{x})=( & \sum_{\lambda \in S_{\beta}} a_{\lambda} B_{\lambda}\left(\sigma_{i} \mathbf{x}\right)\left(\mathcal{P}_{\beta}+\mathcal{Q}_{\sigma_{i} \bullet \beta}\right) \\
& \left.\quad+\sum_{\mu \in S_{\sigma_{i}} \bullet \beta} a_{\mu} B_{\mu}\left(\sigma_{i} \mathbf{x}\right)\left(\mathcal{P}_{\sigma_{i} \bullet \beta}+\mathcal{Q}_{\beta}\right)\right) /\left(1-q^{-m-1} x_{i}^{-m}\right),
\end{aligned}
$$

where we have written $B_{\lambda}(\mathbf{x})=\mathbf{x}^{\lambda}$ and have eliminated the $\ell, i$ from the subscripts to $\mathcal{P}, \mathcal{Q}$ to lighten the notation.

Now going from $F_{\beta, i}$ to $f_{\beta, i}$ is achieved by multiplying the terms in $F_{\beta, i}$ in $S_{\sigma_{i}} \bullet \beta$ by $-\gamma(-\delta)\left(p x_{i}\right)^{m-\delta}$. Thus from (3.34) we have

$$
f_{\beta, i}(\mathbf{x})=\left(\sum_{\lambda \in S_{\beta}} a_{\lambda} \mathbf{x}^{\lambda}-\gamma(-\delta)\left(q x_{i}\right)^{m-\delta} \sum_{\mu \in S_{\sigma_{i}} \bullet \beta} a_{\mu} \mathbf{x}^{\mu}\right) /\left(1-q^{m-1} x_{i}^{m}\right),
$$

which implies that

$$
\begin{aligned}
& f_{\beta, i}\left(\sigma_{i} \mathbf{x}\right) \\
= & \left(\sum_{\lambda \in S_{\beta}} a_{\lambda} B_{\lambda}\left(\sigma_{i} \mathbf{x}\right)-\gamma(-\delta)\left(q x_{i}\right)^{\delta-m} \sum_{\mu \in S_{\sigma_{i} \bullet \beta}} a_{\mu} B_{\mu}\left(\sigma_{i} \mathbf{x}\right)\right) /\left(1-q^{-m-1} x_{i}^{-m}\right) .
\end{aligned}
$$

But (3.35) also equals $F_{\beta, i}(\mathbf{x})$, and there the terms in $S_{\beta}$ (respectively, $S_{\sigma_{i} \bullet \beta}$ ) are those that are multiplied by $\mathcal{P}_{\beta}, \mathcal{Q}_{\beta}$ (resp., $\mathcal{P}_{\sigma_{i} \bullet \beta}, \mathcal{Q}_{\sigma_{i} \bullet \beta}$ ). Hence

$$
\begin{aligned}
& f_{\beta, i}(\mathbf{x})=\left(\sum_{\lambda \in S_{\beta}} a_{\lambda} B_{\lambda}\left(\sigma_{i} \mathbf{x}\right)\left(\mathcal{P}_{\beta}-\gamma(-\delta)\left(q x_{i}\right)^{m-\delta} \mathcal{Q}_{\sigma_{i} \bullet \beta}\right)\right. \\
& \left.+\sum_{\mu \in S_{\sigma_{i} \bullet \beta}} a_{\mu} B_{\mu}\left(\sigma_{i} \mathbf{x}\right)\left(-\gamma(-\delta)\left(q x_{i}\right)^{m-\delta} \mathcal{P}_{\sigma_{i} \bullet \beta}+\mathcal{Q}_{\beta}\right)\right) /\left(1-q^{-m-1} x_{i}^{-m}\right) .
\end{aligned}
$$

Comparing (3.36) and (3.37), we see that $f_{\beta, i}(\mathbf{x}) / f_{\beta, i}\left(\sigma_{i} \mathbf{x}\right)$ equals either of

$$
\mathcal{P}_{\beta}-\gamma(-\delta)\left(q x_{i}\right)^{m-\delta} \mathcal{Q}_{\sigma_{i} \bullet \beta}, \quad\left(q x_{i}\right)^{2(m-\delta)} \mathcal{P}_{\sigma_{i} \bullet \beta}-\mathcal{Q}_{\beta}\left(q x_{i}\right)^{m-\delta} / \gamma(-\delta),
$$

both of which equal $\left(q x_{i}\right)^{l_{i}+1-\delta}$. This completes the proof of the theorem. 


\section{The COEFFicients $H(\mathbf{c} ; \mathbf{m})$}

In this section we explain how $N(\mathbf{x} ; \ell)$ will be used to construct the factor $H(\mathbf{c} ; \mathbf{m})$ that will later be used to define our multiple Dirichlet series (6.1). Henceforth, the constants $\gamma(i)$ used in the definition of the group action in the last section will be specialized to be the modified Gauss sums

$$
\gamma(i)= \begin{cases}g\left(1, \varpi ; \epsilon^{k}\right) / q & \text { if } k \text { is not congruent to } 0 \bmod n, \\ -1 & \text { otherwise. }\end{cases}
$$

Denote the $x_{1}^{\beta_{1}} \cdots x_{r}^{\beta_{r}}$ coefficient of the polynomial $N(\mathbf{x} ; \ell)$ by

$$
H\left(\varpi^{\beta_{1}}, \ldots, \varpi^{\beta_{r}} ; \varpi^{l_{1}}, \ldots, \varpi^{l_{r}}\right) .
$$

To complete the definition of $H$ we impose a twisted multiplicativity property on its coefficients. For fixed $\left(c_{1} \cdots c_{r}, c_{1}^{\prime} \cdots c_{r}^{\prime}\right)=1$, we put

$$
H\left(c_{1} c_{1}^{\prime}, \ldots, c_{r} c_{r}^{\prime} ; \mathbf{m}\right)=\xi\left(\mathbf{c}, \mathbf{c}^{\prime}\right) H\left(c_{1}, \ldots, c_{r} ; \mathbf{m}\right) H\left(c_{1}^{\prime}, \ldots, c_{r}^{\prime} ; \mathbf{m}\right),
$$

where

$$
\xi\left(\mathbf{c}, \mathbf{c}^{\prime}\right)=\prod_{i=1}^{r}\left(\frac{c_{i}}{c_{i}^{\prime}}\right)^{\left\|\alpha_{i}\right\|^{2}}\left(\frac{c_{i}^{\prime}}{c_{i}}\right)^{\left\|\alpha_{i}\right\|^{2}} \prod_{i<j}\left(\frac{c_{i}}{c_{j}^{\prime}}\right)^{2\left\langle\alpha_{i}, \alpha_{j}\right\rangle} \prod_{i<j}\left(\frac{c_{i}^{\prime}}{c_{j}}\right)^{2\left\langle\alpha_{i}, \alpha_{j}\right\rangle} .
$$

We also have the relation

$$
H\left(c_{1}, \ldots, c_{r} ; m_{1} m_{1}^{\prime}, \ldots, m_{r} m_{r}^{\prime}\right)=\prod_{j=1}^{r}\left(\frac{m_{j}^{\prime}}{c_{j}}\right)^{-\left\|\alpha_{j}\right\|^{2}} H\left(c_{1}, \ldots, c_{r} ; m_{1}, \ldots, m_{r}\right)
$$

if $\left(c_{1} \cdots c_{r}, m_{1}^{\prime} \cdots m_{r}^{\prime}\right)=1$. Hence, using properties (4.3) and (4.5) with (4.2), we define $H(\mathbf{c} ; \mathbf{m})$ for any $r$-tuples of integers $\mathbf{c}, \mathbf{m}$ in $\mathcal{O}_{S}$.

For later arguments it is convenient to state Theorem 3.6 in different notation. Given an $r$-tuple of nonnegative integers $\mathbf{k}=\left(k_{1}, \ldots, k_{r}\right)$, define the one-variable polynomials

$$
\begin{aligned}
N^{(\varpi ; \mathbf{k})} & \left(x ; \mathbf{m}, \alpha_{i}\right) \\
& =\sum_{j \geq 0} H\left(\varpi^{k_{1}}, \ldots, \varpi^{k_{i-1}}, \varpi^{j m+\left(k_{i}\right)_{m}}, \varpi^{k_{i+1}}, \ldots, \varpi^{k_{r}} ; \mathbf{m}\right) x^{j m+\left(k_{i}\right)_{m}},
\end{aligned}
$$

where $m=m\left(\alpha_{i}\right)$. For each component $m_{i}$ of $\mathbf{m}$, let $l_{i}=\operatorname{ord}_{\varpi} m_{i}$. Define

$$
\begin{aligned}
& f^{(\varpi ; \mathbf{k})}\left(x ; \mathbf{m}, \alpha_{i}\right)=\frac{N^{(\varpi ; \mathbf{k})}\left(x ; \mathbf{m}, \alpha_{i}\right)}{1-|\varpi|^{m-1} x^{m}} \\
&-\delta_{k_{i}, k_{i}^{\prime}}^{m} g\left(m_{i}^{-1} \varpi^{l_{i}}, \varpi ; \epsilon^{\left\|\alpha_{i}\right\|^{2}\left(k_{i}-k_{i}^{\prime}\right)}\right) q^{\left(k_{i}-k_{i}^{\prime}-1\right)_{m}} x^{\left(k_{i}-k_{i}^{\prime}\right)_{m}} \frac{N^{\left(\varpi ; \mathbf{k}^{\prime}\right)}\left(x ; \mathbf{m}, \alpha_{i}\right)}{1-|\varpi|^{m-1} x^{m}},
\end{aligned}
$$

where $\mathbf{k}^{\prime}$ is defined by

$$
\mathbf{k}^{\prime}=\left(k_{1}, \ldots, k_{i-1},-k_{i}+l_{i}+1-\sum_{j \neq i} k_{j} c(j, i), k_{i+1}, \ldots, k_{r}\right),
$$

and $\delta_{i, j}^{m}$ is 0 if $i \equiv j(\bmod m)$ and 1 otherwise. Note that if $\beta \in \Lambda$ is written as $\beta=\sum k_{i} \alpha_{i}$, where the $\alpha_{i}$ are the simple roots, then $\sigma_{i} \bullet \beta$ is $\sum k_{i}^{\prime} \alpha_{i}$. Moreover, if $\mathbf{m}$ consists of pure $\varpi$-powers, then the polynomial $N^{(\varpi ; \mathbf{k})}$ (respectively, $f^{(\varpi ; \mathbf{k})}$ ) coincides with $N_{\beta, i}$ (resp., $f_{\beta, i}$ ) from the end of Section 3 after setting $x_{j}=1$ for all $j \neq i$. Then a mild generalization of Theorem 3.6 yields the following: 
Theorem 4.1. We have

$$
\frac{f^{(\varpi ; \mathbf{k})}\left(x ; \mathbf{m}, \alpha_{i}\right)}{f^{(\varpi ; \mathbf{k})}\left(1 /\left(q^{2} x\right) ; \mathbf{m}, \alpha_{i}\right)}= \begin{cases}(q x)^{l_{i}+1-\left(k_{i}^{\prime}-k_{i}\right)_{m}} & \text { if }\left(k_{i}^{\prime}-k_{i}\right)_{m} \neq 0, \\ (q x)^{l_{i}+1-m} & \text { otherwise. }\end{cases}
$$

\section{Kubota's Dirichlet SERIES}

In this section we define and describe the functional equations of the Kubota Dirichlet series. These are Dirichlet series in one complex variable whose coefficients are $n$ th-order Gauss sums. These series arise as the Whittaker coefficients of an Eisenstein series on the $n$-fold metaplectic cover of $G L_{2}(F)$. For an integer $j$, a function $\Psi \in \mathcal{M}_{j}(\Omega)$, and $a \in \mathcal{O}_{S}^{\times}$, we define

$$
\mathcal{D}\left(s, a ; \Psi, \epsilon^{j}\right)=\sum_{0 \neq c \in \mathcal{O}_{S} / \mathcal{O}_{S}^{\times}} \frac{g\left(a, c ; \epsilon^{j}\right) \Psi(c)}{|c|^{s}} .
$$

This is initially defined for $\operatorname{Re}(s)>3 / 2$, where the series is absolutely convergent.

Let $m=\operatorname{gcd}(n, j)$ and set

$$
G_{m}(s)=\left((2 \pi)^{-(m-1)(s-1)} \Gamma(m s-m) / \Gamma(s-1)\right)^{[F: \mathbb{Q}] / 2} .
$$

Define

$$
\mathcal{D}^{*}\left(s, a ; \Psi, \epsilon^{j}\right)=G_{m}(s) \zeta(m s-m+1) \mathcal{D}\left(s, a ; \Psi, \epsilon^{j}\right),
$$

where $\zeta$ is the Dedekind zeta function of $F$.

If $\Psi \in \mathcal{M}_{j}(\Omega)$ and $\eta \in F^{\times}$we define

$$
\hat{\Psi}_{\eta}(c)=(\eta, c)^{j} \Psi(\eta c) \quad \text { and } \quad \tilde{\Psi}_{\eta}(c)=(\eta, c)^{j} \Psi\left(\eta^{-1} c^{-1}\right) .
$$

It is easy to see that $\hat{\Psi}_{\eta}$ and $\tilde{\Psi}_{\eta}$ are both in $\mathcal{M}_{j}(\Omega)$ and that they depend only on the class of $\eta$ in $F_{S}^{\times} / F_{S}^{\times n}$, where the $n$ denotes taking $n$th powers. Then we have the following:

Proposition 5.1 ([Kub71b, Kub71a, BB06a]). The function $\mathcal{D}^{*}\left(s, a ; \Psi, \epsilon^{j}\right)$ has a meromorphic continuation to $\mathbb{C}$ and is holomorphic except for possible simple poles at $s=1 \pm 1 / m$. Moreover $\mathcal{D}^{*}\left(s, a ; \Psi, \epsilon^{j}\right)$ satisfies the functional equation

$$
\mathcal{D}^{*}\left(s, a ; \Psi, \epsilon^{j}\right)=|a|^{1-s} \sum_{\eta \in F_{S}^{\times} / F_{S}^{\times n}} P_{a \eta}^{j}(s) \mathcal{D}^{*}\left(2-s, a ; \tilde{\Psi}_{\eta}, \epsilon^{j}\right) .
$$

Here the $P_{a \eta}^{j}(s)$ are Dirichlet polynomials supported on the places in $S$.

Remark 5.2. Based on the explicit functional equations given in the function field case (see Pat07, Hof92]), we expect the Dirichlet polynomials to be closely related to the rational functions $\mathcal{P}$ and $\mathcal{Q}$ which appear in Section 3. However, the nature of the precise relationship is unclear and not needed for what follows.

Given a set of primes $T$, we define

$$
\mathcal{D}_{T}\left(s, a ; \Psi, \epsilon^{j}\right)=\sum_{\substack{0 \neq c \in \mathcal{O}_{S} / \mathcal{O}_{S}^{\times} \\(c, T)=1}} \frac{g\left(a, c ; \epsilon^{j}\right) \Psi(c)}{|c|^{s}} .
$$

If $\mathfrak{m}_{0}=\prod_{\varpi \in T} \varpi$ we sometimes write $\mathcal{D}_{\mathfrak{m}_{0}}\left(s, a ; \Psi, \epsilon^{j}\right)$ for $\mathcal{D}_{T}\left(s, a ; \Psi, \epsilon^{j}\right)$.

Using properties of Gauss sums, we can relate the functions $\mathcal{D}_{T}$ to the functions $\mathcal{D}_{T^{\prime}}$ for different sets $T$ and $T^{\prime}$. This is the content of the following two lemmas. 
Lemma 5.3. Let $\varpi \in \mathcal{O}_{S} / \mathcal{O}_{S}^{\times}$be prime of norm $q$. For an integer $i$ with $0 \leq i \leq$ $m-1$ and $a_{1}, a_{2}, \varpi$ all pairwise relatively prime, we have

$$
\begin{aligned}
& \mathcal{D}_{a_{1}}\left(s, a_{2} \varpi^{i} ; \Psi, \epsilon^{j}\right) \\
= & \mathcal{D}_{\varpi a_{1}}\left(s, a_{2} \varpi^{i} ; \Psi, \epsilon^{j}\right)+\frac{g\left(a_{2} \varpi^{i}, \varpi^{i+1} ; \epsilon^{j}\right)}{q^{(i+1) s}} \mathcal{D}_{\varpi a_{1}}\left(s, a_{2} \varpi^{(m-i-2)_{m}} ; \hat{\Psi}_{\eta}, \epsilon^{j}\right),
\end{aligned}
$$

where $\eta \sim \varpi^{i+1}$. Here we write $a \sim b$ to mean that $a, b$ lie in the same coset modulo $F_{S}^{\times n}$.

Proof. For $\varpi, a_{1}, a_{2}$ as in the statement,

$$
\begin{aligned}
\mathcal{D}_{a_{1}}\left(s, a_{2} \varpi^{i} ; \Psi, \epsilon^{j}\right) \\
=\sum_{\substack{0 \neq c \in \mathcal{O}_{S} / \mathcal{O}_{S}^{\times} \\
\left(c, a_{1}\right)=1}} \frac{g\left(a_{2} \varpi^{i}, c ; \epsilon^{j}\right) \Psi(c)}{|c|^{s}} \\
=\sum_{k \geq 0} \sum_{\left(c, a_{1} \varpi\right)=1} \frac{g\left(a_{2} \varpi^{i}, c \varpi^{k} ; \epsilon^{j}\right) \Psi\left(q^{k} c\right)}{|c|^{s} q^{k s}} \\
=\sum_{k \geq 0} \sum_{\left(c, a_{1} \varpi\right)=1} \frac{g\left(a_{2} \varpi^{i}, c ; \epsilon^{j}\right) g\left(a_{2} \varpi^{i}, \varpi^{k} ; \epsilon^{j}\right) \Psi\left(q^{k} c\right)}{|c|^{s} q^{k s}}\left(\frac{c}{\varpi^{k}}\right)^{j}\left(\frac{\varpi^{k}}{c}\right)^{j} \\
=\sum_{\left(c, a_{1} \varpi\right)=1} \frac{g\left(a_{2} \varpi^{i}, c ; \epsilon^{j}\right)}{|c|^{s}}\left(\sum_{k \geq 0} \frac{g\left(a_{2} \varpi^{i}, \varpi^{k} ; \epsilon^{j}\right) \Psi\left(q^{k} c\right)}{q^{k s}}\left(\frac{\varpi^{2 j k}}{c}\right)\left(\varpi^{k}, c\right)_{S}^{j}\right) .
\end{aligned}
$$

The Gauss sum in the inner sum vanishes unless $k=0$ or $i+1$. This proves the lemma.

Inverting the previous lemma, we obtain

Lemma 5.4. If $0 \leq i \leq m-2$ and $a_{1}, a_{2}$, $\varpi$ are as above, and $\eta \sim \varpi^{i+1}$, then

$$
\begin{aligned}
\mathcal{D}_{\varpi a_{1}}( & \left.s, a_{2} \varpi^{i} ; \Psi, \epsilon^{j}\right) \\
& =\frac{\mathcal{D}_{a_{1}}\left(s, a_{2} \varpi^{i} ; \Psi, \epsilon^{j}\right)}{1-|\varpi|^{m-1-m s}}-\frac{g\left(a_{2} \varpi^{i}, \varpi^{i+1} ; \epsilon^{j}\right)}{|\varpi|^{(i+1) s}} \frac{\mathcal{D}_{a_{1}}\left(s, a_{2} \varpi^{m-i-2} ; \hat{\Psi}_{\eta}, \epsilon^{j}\right)}{1-|\varpi|^{m-1-m s}},
\end{aligned}
$$

and if $i=m-1$, then

$$
\mathcal{D}_{\varpi a_{1}}\left(s, a_{2} \varpi^{i} ; \Psi, \epsilon^{j}\right)=\frac{\mathcal{D}_{a_{1}}\left(s, a_{2} \varpi^{i} ; \Psi, \epsilon^{j}\right)}{1-|\varpi|^{m-1-m s}} .
$$

Before stating and proving the main result of this section, we need to extend the functional equation of the Kubota series $\mathcal{D}\left(s, a ; \Psi, \epsilon^{j}\right)$ to a slightly more general class of $\Psi$. We follow Section 5 of [BBF06] and Section 7 of [BBF08. Let $\mathbf{m}=$ $\left(m_{1}, \ldots, m_{r}\right)$. This $r$-tuple will be fixed for the rest of the section. Let $\mathcal{M}(\Omega, \Phi)$ be the space of functions $\Psi:\left(F_{S}^{\times}\right)^{r} \rightarrow \mathbb{C}$ such that

$$
\Psi\left(\varepsilon_{1} a_{1}, \ldots, \varepsilon_{r} a_{r}\right)=\prod_{i=1}^{r}\left(\varepsilon_{i}, a_{i}\right)_{S}^{\left\|\alpha_{i}\right\|^{2}}\left(\prod_{i<j}\left(\varepsilon_{i}, a_{j}\right)_{S}^{2\left\langle\alpha_{i}, \alpha_{j}\right\rangle}\right) \Psi\left(a_{1}, \ldots, a_{r}\right) .
$$

Let $\mathcal{A}$ be the ring of Laurent polynomials in $\left|\varpi_{v}\right|^{s_{i}}$, where $v$ runs over the places in $S_{\text {fin }}$. Define $\mathfrak{M}=\mathfrak{M}(\Omega, \Phi)=\mathcal{A} \otimes \mathcal{M}(\Omega, \Phi)$ and $\mathfrak{M}_{j}(\omega)=\mathcal{A} \otimes \mathcal{M}_{j}(\Omega)$. If $\Psi \in$ 
$\mathfrak{M}(\Omega, \Phi)$ and $a_{1}, \ldots, a_{r} \in \mathcal{O}_{S} / \mathcal{O}_{S}^{\times}$, define $\Psi_{i}^{\left(a_{1}, \ldots, a_{r}\right)}$ by

$$
\Psi_{i}^{\left(a_{1}, \ldots, a_{r}\right)}(\mathbf{s} ; c)=\Psi\left(\mathbf{s} ; a_{1}, \ldots, a_{i} c, \ldots, a_{r}\right)\left(c, a_{i}\right)_{S}^{-\left\|\alpha_{i}\right\|^{2}} \prod_{j>i}\left(c, a_{j}\right)_{S}^{-2\left\langle\alpha_{i}, \alpha_{j}\right\rangle} .
$$

Lemma 5.5 (Lemma 5.3 [BBF06]). For fixed $a_{1}, \ldots, a_{r} \in \mathcal{O}_{S} / \mathcal{O}_{S}^{\times}$, we have

$$
\Psi_{i}^{\left(a_{1}, \ldots, a_{r}\right)} \in \mathfrak{M}_{\left\|\alpha_{i}\right\|^{2}}(\Omega) .
$$

Continuing to follow BBF06, BBF08, we define an action of the Weyl group $W$ on $\mathfrak{M}(\Omega, \Phi)$. First, we let the simple reflection $\sigma_{i}$ act on the complex vector $\mathbf{s}$ by

$$
\left(\sigma_{i} \mathbf{s}\right)_{j}=s_{j}-c(j, i)\left(s_{i}-1\right),
$$

where $c(j, i)$ is the Cartan integer. This corresponds to the action (3.8) under the change of variables $x_{i}=q^{-s_{i}}$. Then for the simple reflection $\sigma_{i}$ and $\Psi \in \mathfrak{M}(\Omega, \Phi)$, define

$$
\begin{aligned}
\left(\sigma_{i} \Psi\right)\left(\mathbf{s} ; a_{1}, \ldots, a_{r}\right)= & \left(\sum_{\eta \in F_{S}^{\times} / F_{S}^{\times n}} P_{\eta m_{i} b_{i}}^{\left\|\alpha_{i}\right\|^{2}}\left(s_{i}\right)\left(\eta, a_{i}\right)_{S}^{\left\|\alpha_{i}\right\|^{2}} \prod_{j>i}\left(\eta, a_{j}\right)_{S}^{2\left\langle\alpha_{i}, \alpha_{j}\right\rangle}\right) \\
& \times \Psi\left(\sigma_{i} \mathbf{s} ; a_{1}, \ldots, a_{r}\right),
\end{aligned}
$$

where $b_{i}=\prod_{i} a_{i}^{-2\left\langle\alpha_{i}, \alpha_{j}\right\rangle /\left\langle\alpha_{i}, \alpha_{i}\right\rangle}$ and $\mathbf{s}=\left(s_{1}, \ldots, s_{r}\right)$. The $W$-action on $\mathfrak{M}(\Omega, \Phi)$ depends on the twisting parameter $\mathbf{m}$, but we suppress this dependence from the notation. When there is no chance of confusion, we will not explicitly indicate the s-dependence for $\Psi$ in $\mathfrak{M}(\Omega, \Phi)$ or $\mathfrak{M}_{j}(\Omega)$.

Proposition 5.6 (Proposition 5.6 [BBF06]). If $\Psi \in \mathfrak{M}(\Omega, \Phi)$, then $\sigma_{i} \Psi \in \mathfrak{M}(\Omega, \Phi)$.

We can now restate the functional equation of Proposition 5.1]in a slightly cleaner form.

Proposition 5.7 (Lemma 5 [BBF08]). Given $\Psi \in \mathfrak{M}(\Omega, \Phi)$ we have

$$
\begin{aligned}
& \mathcal{D}^{*}\left(s_{i}, m_{i} b_{i} ; \Psi_{i}^{\left(a_{1}, \ldots, a_{r}\right)}, \epsilon^{\left\|\alpha_{i}\right\|^{2}}\right) \\
&=\left|m_{i} b_{i}\right|^{1-s_{i}} \mathcal{D}^{*}\left(2-s_{i}, m_{i} b_{i} ;\left(\sigma_{i} \Psi\right)_{i}^{\left(a_{1}, \ldots, a_{r}\right)}, \epsilon^{\left\|\alpha_{i}\right\|^{2}}\right)
\end{aligned}
$$

for $b_{i}=\prod_{i} a_{i}^{-2\left\langle\alpha_{i}, \alpha_{j}\right\rangle /\left\langle\alpha_{i}, \alpha_{i}\right\rangle}$.

We now turn to the main result of this section. Let a be the $(r-1)$-tuple $\left(a_{1}, \ldots, \hat{a}_{i}, \ldots, a_{r}\right)$, where the hat on $a_{i}$ indicates that this entry is omitted. Let $\Psi \in \mathfrak{M}(\Omega, \Phi)$. Introduce the Dirichlet series

$$
\mathcal{E}\left(s_{i}, \mathbf{a} ; \mathbf{m}, \Psi, i\right)=\sum_{0 \neq a_{i} \in \mathcal{O}_{S} / \mathcal{O}_{S}^{\times}} \frac{H\left(a_{1}, \ldots, a_{i}, \ldots, a_{r} ; \mathbf{m}\right) \Psi\left(a_{1}, \ldots, a_{i}, \ldots, a_{r}\right)}{\left|a_{i}\right|^{S_{i}}} .
$$

Let $m=m(\alpha)$ and define

$$
\mathcal{E}^{*}\left(s_{i}, \mathbf{a} ; \mathbf{m}, \Psi, i\right)=\zeta\left(m s_{i}-m+1\right) G_{m}\left(s_{i}\right) \mathcal{E}\left(s_{i}, \mathbf{a} ; \mathbf{m}, \Psi, i\right) .
$$

Theorem 5.8. Let $A=\prod_{j \neq i} a_{j}^{-2\left\langle\alpha_{i}, \alpha_{j}\right\rangle /\left\|\alpha_{i}\right\|^{2}}$. Then

$$
\mathcal{E}^{*}\left(s_{i}, \mathbf{a} ; \mathbf{m}, \Psi, i\right)=\left|A m_{i}\right|^{1-s_{i}} \mathcal{E}^{*}\left(2-s_{i}, \mathbf{a} ; \mathbf{m}, \sigma_{i} \Psi, i\right) .
$$


Proof. To simplify notation, assume that $i=1$. Let $\varpi_{1}, \ldots, \varpi_{V}$ be the prime divisors of $a_{2} \cdots a_{r} m_{1} \cdots m_{r}$, with $\left|\varpi_{j}\right|=q_{j}$. Let $T=\left\{\varpi_{1}, \ldots, \varpi_{V}\right\}$. Write $a_{j}=$ $\varpi_{1}^{\beta_{j 1}} \cdots \varpi_{V}^{\beta_{j V}}$ for $j=2, \ldots, r$ and $A m_{1}=\varpi_{1}^{l_{1}} \ldots \varpi_{V}^{l_{V}}$. If $a_{1}$ has no divisors in $T$ and $a_{1}^{\prime}=\varpi_{1}^{\beta_{11}} \cdots \varpi_{V}^{\beta_{1 V}}$, we expand the $H$-coefficient

$$
\begin{aligned}
& H\left(a_{1} a_{1}^{\prime}, a_{2}, \ldots, a_{r} ; \mathbf{m}\right)=\left(\frac{a_{1}^{\prime}}{a_{1}}\right)^{2\left\|\alpha_{1}\right\|^{2}}\left(\frac{A}{a_{1}}\right)^{-\left\|\alpha_{1}\right\|^{2}}\left(a_{1}^{\prime}, a_{1}\right)_{S}^{\left\|\alpha_{1}\right\|^{2}}\left(A, a_{1}\right)_{S}^{-\left\|\alpha_{1}\right\|^{2}} \\
& \quad \times H\left(a_{1}, 1, \ldots, 1 ; \mathbf{m}\right) H\left(\varpi_{1}^{\beta_{11}} \cdots \varpi_{V}^{\beta_{1 V}}, \varpi_{1}^{\beta_{21}} \cdots \varpi_{V}^{\beta_{2 V}}, \ldots, \varpi_{1}^{\beta_{r 1}} \cdots \varpi_{V}^{\beta_{r V}} ; \mathbf{m}\right) .
\end{aligned}
$$

By the multiplicativity relation (4.5) and Theorem 3.5, the first $H$-coefficient on the right-hand side is

$$
H\left(a_{1}, 1, \ldots, 1 ; \mathbf{m}\right)=g\left(1, a_{1} ; \epsilon^{\left\|\alpha_{1}\right\|^{2}}\right)\left(\frac{m_{1}}{a_{1}}\right)^{-\left\|\alpha_{1}\right\|^{2}} .
$$

We further expand the second $H$-coefficient on the right-hand side as

$$
\begin{aligned}
& H\left(\varpi_{1}^{\beta_{11}} \cdots \varpi_{V}^{\beta_{1 V}}, \varpi_{1}^{\beta_{21}} \cdots \varpi_{V}^{\beta_{2 V}}, \ldots, \varpi_{1}^{\beta_{r 1}} \cdots \varpi_{V}^{\beta_{r V}} ; \mathbf{m}\right) \\
& \quad=\left(\prod_{\substack{1 \leq j \leq r \\
i \neq i^{\prime}}}\left(\frac{\varpi_{i}^{\beta_{j i}}}{\varpi_{i^{\prime}}^{\beta_{j^{\prime}}}}\right)^{\left\|\alpha_{j}\right\|^{2}}\right)\left(\prod_{\substack{j<j^{\prime} \\
i \neq i^{\prime}}}\left(\frac{\varpi_{i}^{\beta_{j i}}}{\varpi_{i^{\prime}}^{\beta_{j^{\prime} i^{\prime}}}}\right)^{2\left\langle\alpha_{j}, \alpha_{j^{\prime}}\right\rangle}\right) \prod_{i=1}^{V} H\left(\varpi_{i}^{\beta_{1 i}}, \ldots, \varpi_{i}^{\beta_{r i}} ; \mathbf{m}\right) .
\end{aligned}
$$

Therefore, up to a multiplicative factor of norm 1 coming from a product of power residue symbols, $\mathcal{E}(s, \mathbf{a} ; \mathbf{m}, \Psi, 1)$ is equal to

$$
\begin{aligned}
& \sum_{\substack{(a, T)=1 \\
k_{1}, \ldots, k_{V} \geq 0}} \frac{g\left(1, a ; \epsilon^{\left\|\alpha_{1}\right\|^{2}}\right)}{|a|^{s_{1}} q_{1}^{k_{1} s_{1}} \cdots q_{V}^{k_{V} s_{1}}}\left(\frac{\varpi_{1}^{2 k_{1}} \cdots \varpi_{V}^{2 k_{V}} A^{-1} m_{1}^{-1}}{a}\right)^{\left\|\alpha_{1}\right\|^{2}} \Psi_{1}^{\left(\varpi_{1}^{k_{1}} \ldots \varpi_{V}^{k_{V}}, a_{2}, \ldots, a_{r}\right)}\left(a_{1}\right) \\
& \quad \times\left(\prod_{i \neq i^{\prime}}\left(\frac{\varpi_{i}^{k_{i}}}{\varpi_{i^{\prime}}^{k_{i^{\prime}}}}\right)^{\left\|\alpha_{1}\right\|^{2}}\right)\left(\prod_{\substack{2 \leq j \leq r \\
i \neq i^{\prime}}}\left(\frac{\varpi_{i}^{k_{i}}}{\varpi_{i^{\prime}}^{\beta_{i^{\prime}}}}\right)^{2\left\langle\alpha_{1}, \alpha_{j}\right\rangle}\right) \prod_{i=1}^{V} H\left(\varpi_{i}^{k_{i}}, \varpi_{i}^{\beta_{2 i}}, \ldots, \varpi_{i}^{\beta_{r i}} ; \mathbf{m}\right) \\
& =\sum_{k_{1}, \ldots, k_{V}=0}^{m-1} \mathcal{D}_{T}\left(s_{1}, \varpi_{1}^{\left(l_{1}-2 k_{1}\right)_{m}} \cdots \varpi_{V}^{\left(l_{V}-2 k_{V}\right)_{m}} ; \Psi_{1}^{\left(\varpi_{1}^{k_{1}} \cdots \varpi_{V}^{k_{V}}, a_{2}, \ldots, a_{r}\right)}, \epsilon^{\left\|\alpha_{1}\right\|^{2}}\right) \\
& \quad \times \prod_{i=1}^{V} N^{\left(\varpi_{i} ; k_{i}, \beta_{2 i}, \ldots, \beta_{r i}\right)}\left(q_{i}^{-s_{1}} ; \mathbf{m}, \alpha_{1}\right)\left(\prod_{i \neq i^{\prime}}\left(\frac{\varpi_{i}^{k_{i}}}{\varpi_{i^{\prime}}^{k_{i^{\prime}}}}\right)^{\left\|\alpha_{1}\right\|^{2}}\right)\left(\prod_{\substack{2 \leq j \leq r \\
i \neq i^{\prime}}}\left(\frac{\varpi_{i}^{\varpi_{i}}}{\varpi_{i^{\prime}}^{\beta_{j i^{\prime}}}}\right)^{2\left\langle\alpha_{1}, \alpha_{j}\right\rangle}\right) .
\end{aligned}
$$

Let $\theta^{\left(\varpi_{1}^{k_{1}}\right)}=\Psi_{1}^{\left(\varpi_{1}^{k_{1}} \ldots \varpi_{V}^{k_{V}}, a_{2}, \ldots, a_{r}\right)}$ and denote by $C\left(k_{1}\right)=C\left(k_{1}, \ldots, k_{r}\right)$ the product of residue symbols

$$
\left(\prod_{i \neq i^{\prime}}\left(\frac{\varpi_{i}^{k_{i}}}{\varpi_{i^{\prime}}^{k_{i^{\prime}}}}\right)^{\left\|\alpha_{1}\right\|^{2}}\right)\left(\prod_{\substack{2 \leq j \leq r \\ i \neq i^{\prime}}}\left(\frac{\varpi_{i}^{k_{i}}}{\varpi_{i^{\prime}}^{\beta_{i^{\prime}}}}\right)^{2\left\langle\alpha_{1}, \alpha_{j}\right\rangle}\right) .
$$


Letting $K_{i}=\left(l_{i}-2 k_{i}\right)_{m}$ for $i=1, \ldots, r$, we have by Lemma 5.4

$$
\begin{aligned}
& \left(1-\left|\varpi_{1}\right|^{m-1-m s_{1}}\right) \mathcal{D}_{T}\left(s_{1}, \varpi_{1}^{K_{1}} \cdots \varpi_{V}^{K_{V}} ; \theta^{\left(\varpi_{1}^{k_{1}}\right)}, \epsilon^{\left\|\alpha_{1}\right\|^{2}}\right) C\left(k_{1}\right) \\
& =\mathcal{D}_{T-\left\{\varpi_{1}\right\}}\left(s_{1}, \varpi_{1}^{K_{1}} \cdots \varpi_{V}^{K_{V}} ; \theta^{\left(\varpi_{1}^{k_{1}}\right)}, \epsilon^{\left\|\alpha_{1}\right\|^{2}}\right) C\left(k_{1}\right) \\
& -\delta_{K_{1},-1}^{m} \frac{g\left(\varpi_{1}^{K_{1}} \cdots \varpi_{V}^{K_{V}}, \varpi_{1}^{K_{1}+1} ; \epsilon^{\left\|\alpha_{1}\right\|^{2}}\right)}{q_{1}^{\left(K_{1}+1\right) s_{1}}} \\
& \quad \times \mathcal{D}_{T-\left\{\varpi_{1}\right\}}\left(s_{1}, \varpi_{1}^{\left(2 k_{1}-l_{1}-2\right)_{m}} \cdots \varpi_{V}^{K_{V}} ; \hat{\theta}_{\eta}^{\left(\varpi_{1}^{k_{1}}\right)}, \epsilon^{\left\|\alpha_{1}\right\|^{2}}\right) C\left(k_{1}\right),
\end{aligned}
$$

where $\eta \sim \varpi_{1}^{K_{1}+1}$ and $\delta$ is defined after (4.7). Consider the second term on the right, with $k_{1}$ replaced by $\left(l_{1}+1-k_{1}\right)_{m}$. For $\delta_{K_{1},-1}^{m} \neq 0$ this gives

$$
\begin{array}{r}
\frac{g\left(\varpi_{1}^{\left(2 k_{1}-l_{1}-2\right)_{m}} \varpi_{2}^{K_{2}} \cdots \varpi_{V}^{K_{V}}, \varpi_{1}^{\left(2 k_{1}-l_{1}-1\right)_{m}} ; \epsilon^{\left\|\alpha_{1}\right\|^{2}}\right)}{q_{1}^{\left(2 k_{1}-l_{1}-1\right)_{m} s_{1}}} C\left(l_{1}-k_{1}+1\right) \\
\times \mathcal{D}_{T-\left\{\varpi_{1}\right\}}\left(s_{1}, \varpi_{1}^{K_{1}} \cdots \varpi_{V}^{K_{V}} ; \hat{\theta}_{\eta^{\prime}}^{\left(\varpi_{1}^{\left(l_{1}+1-k_{1}\right)_{m}}\right)}, \epsilon^{\left\|\alpha_{1}\right\|^{2}}\right),
\end{array}
$$

where $\eta^{\prime} \sim \varpi_{1}^{2 k_{1}-l_{1}-1}$.

The Gauss sum can be written as

$$
\left(\frac{\varpi_{2}^{K_{2}} \cdots \varpi_{V}^{K_{V}}}{\varpi_{1}^{2 k_{1}-l_{1}-1}}\right)^{-\left\|\alpha_{1}\right\|^{2}} g\left(\varpi_{1}^{\left(2 k_{1}-l_{1}-2\right)_{m}}, \varpi_{1}^{\left(2 k_{1}-l_{1}-1\right)_{m}} ; \epsilon^{\left\|\alpha_{1}\right\|^{2}}\right) .
$$

Keeping careful track of the Hilbert symbols, we get

Lemma 5.9. For $\eta^{\prime}$ as above,

$$
C\left(k_{1}\right) \theta^{\left(\varpi_{1}^{k_{1}}\right)}=C\left(l_{1}-k_{1}+1\right)\left(\frac{m_{1} \varpi_{2}^{K_{2}} \cdots \varpi_{V}^{K_{V}}}{\varpi_{1}^{2 k_{1}-l_{1}-1}}\right)^{-\left\|\alpha_{1}\right\|^{2}} \hat{\theta}_{\eta^{\prime}}^{\left(\varpi_{1}^{\left(l_{1}+1-k_{1}\right) m}\right)} .
$$

Proof of Lemma [5.9. Define $l_{i}^{\prime}$ by $\prod_{2 \leq j \leq r} \varpi_{i}^{-2\left\langle\alpha_{1}, \alpha_{j}\right\rangle \beta_{j i}}=\varpi_{i}^{l_{i}^{\prime}}$. We have

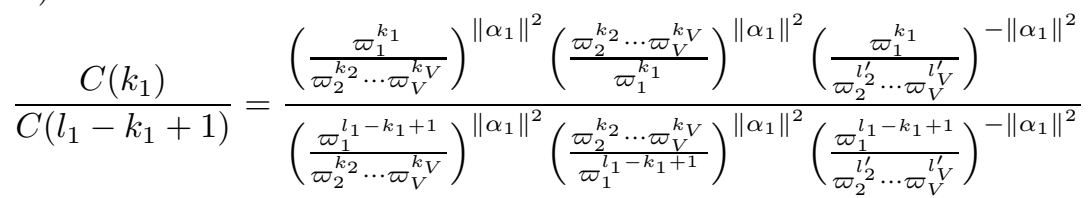

$$
\begin{aligned}
& =\left(\frac{\varpi_{1}^{2 k_{1}-l_{1}-1}}{\varpi_{2}^{2 k_{2}-l_{2}^{\prime}} \cdots \varpi_{V}^{2 k_{V}-l_{V}^{\prime}}}\right)^{\left\|\alpha_{1}\right\|^{2}}\left(\varpi_{1}^{2 k_{1}-l_{1}-1}, \varpi_{2}^{k_{2}} \cdots \varpi_{V}^{k_{V}}\right)^{\left\|\alpha_{1}\right\|^{2}} \\
& =\left(\frac{\varpi_{2}^{2 k_{2}-l_{2}^{\prime}} \cdots \varpi_{V}^{2 k_{V}-l_{V}^{\prime}}}{\varpi_{1}^{2 k_{1}-l_{1}-1}}\right)^{\left\|\alpha_{1}\right\|^{2}}\left(\varpi_{1}^{2 k_{1}-l_{1}-1}, \varpi_{2}^{l_{2}^{\prime}-k_{2}} \cdots \varpi_{V}^{l_{V}^{\prime}-k_{V}}\right)^{\left\|\alpha_{1}\right\|^{2}} .
\end{aligned}
$$

Further, $\theta^{\left(\varpi_{1}^{k_{1}}\right)} / \hat{\theta}_{\eta^{\prime}}^{\left(\varpi_{1}^{\left(l_{1}+1-k_{1}\right) m}\right)}=\left(\varpi_{1}^{2 k_{1}-l_{1}-1}, \varpi_{2}^{k_{2}-l_{2}^{\prime}} \cdots \varpi_{V}^{k_{V}-l_{V}^{\prime}}\right)^{\left\|\alpha_{1}\right\|^{2}}$. Thus

$$
\begin{aligned}
\frac{C\left(k_{1}\right) \theta^{\left(\varpi_{1}^{k_{1}}\right)}}{C\left(l_{1}-k_{1}+1\right) \hat{\theta}_{\eta^{\prime}}^{\left(\varpi_{1}^{\left(l_{1}+1-k_{1}\right) m}\right)}} & =\left(\frac{\varpi_{2}^{2 k_{2}-l_{2}^{\prime}} \cdots \varpi_{V}^{2 k_{V}-l_{V}^{\prime}}}{\varpi_{1}^{2 k_{1}-l_{1}-1}}\right)^{\left\|\alpha_{1}\right\|^{2}} \\
& =\left(\frac{m_{1} \varpi_{2}^{K_{2}} \cdots \varpi_{V}^{K_{V}}}{\varpi_{1}^{2 k_{1}-l_{1}-1}}\right)^{-\left\|\alpha_{1}\right\|^{2}} .
\end{aligned}
$$


We now continue from the last line of (5.12). Use (5.14) to write $\mathcal{D}_{T}$ as a linear combination of two Kubota series of type $\mathcal{D}_{T-\left\{\varpi_{1}\right\}}$ and replace $k_{1}$ by $\left(l_{1}+1-k_{1}\right)_{m}$ in the second of these. Then we use Lemma 5.9 to put the two Kubota series back together and find that, up to a product of residue symbols, $\mathcal{E}\left(s_{1}, \mathbf{a} ; \mathbf{m}, \Psi, 1\right)$ is

$$
\begin{aligned}
& \sum_{k_{1}, \ldots, k_{V}=0}^{m-1} \mathcal{D}_{T-\varpi_{1}}\left(s_{1}, \varpi_{1}^{\left(l_{l}-2 k_{1}\right)_{m}} \cdots \varpi_{V}^{\left(l_{V}-2 k_{V}\right)_{m}} ; \theta^{\left(\varpi_{1}^{k}\right)}, \epsilon^{\left\|\alpha_{1}\right\|^{2}}\right) C\left(k_{1}, \ldots, k_{r}\right) \\
& \quad \times f^{\left(\varpi_{1} ; k_{1}, \beta_{21}, \ldots, \beta_{r 1}\right)}\left(q_{1}^{-s_{1}} ; \mathbf{m}, \alpha_{1}\right) \prod_{i=2}^{V} N^{\left(\varpi_{i} ; k_{i}, \beta_{2 i}, \ldots, \beta_{r i}\right)}\left(q_{i}^{-s_{1}} ; \mathbf{m}, \alpha_{1}\right) .
\end{aligned}
$$

Repeating this process for $\varpi_{2}, \ldots, \varpi_{r}$ in order to remove the remaining primes from $T$, we arrive at

$$
\begin{aligned}
& \mathcal{E}\left(s_{1}, \mathbf{a} ; \mathbf{m}, \Psi, 1\right) \\
& \begin{array}{c}
(5.20)=\xi \sum_{k_{1}, \ldots, k_{V}=0}^{m-1} \mathcal{D}\left(s_{1}, \varpi_{1}^{\left(l_{1}-2 k_{1}\right)_{m}} \cdots \varpi_{V}^{\left(l_{V}-2 k_{V}\right)_{m}} ; \Psi_{1}^{\left(\varpi_{1}^{k_{1}} \ldots \varpi_{V}^{k_{V}}, a_{2}, \ldots, a_{r}\right)}, \epsilon^{\left\|\alpha_{1}\right\|^{2}}\right) \\
\quad \times C\left(k_{1}, \ldots, k_{r}\right) \prod_{i=1}^{V} f^{\left(\varpi_{i} ; k_{i}, \beta_{2 i}, \ldots, \beta_{r i}\right)}\left(q_{i}^{-s_{1}} ; \mathbf{m}, \alpha_{1}\right),
\end{array}
\end{aligned}
$$

for $\xi$ a product of residue symbols. The theorem is now a consequence of the functional equation of Kubota's Dirichlet series given in Proposition 5.7, together with the functional equation of $f$ given in Theorem 4.1.

\section{The multiple Dirichlet Series}

Given $\Psi \in \mathfrak{M}(\Omega, \Phi)$ and $\mathbf{m}=\left(m_{1}, \ldots, m_{r}\right)$ an $r$-tuple of nonzero integers in $\mathcal{O}_{S}$, we define the multiple Dirichlet series in $r$ complex variables:

$$
Z(\mathbf{s} ; \mathbf{m}, \Psi)=\sum_{0 \neq c_{1}, \ldots, c_{r} \in \mathcal{O}_{S} / \mathcal{O}_{S}^{\times}} \frac{H\left(c_{1}, \ldots, c_{r} ; \mathbf{m}\right) \Psi\left(c_{1}, \ldots, c_{r}\right)}{\prod\left|c_{i}\right|^{s_{i}}} .
$$

This is initially defined for $\mathbf{s}=\left(s_{1}, \ldots, s_{n}\right)$ an $r$-tuple of complex numbers with $\operatorname{Re}\left(s_{i}\right)>3 / 2$. To describe the functional equations satisfied by $Z$, we need to introduce some Gamma factors. For any positive root $\alpha \in \Phi^{+}$, write $\alpha=\sum k_{i} \alpha_{i}$ as in (3.5). Define

$$
\zeta_{\alpha}(s)=\zeta\left(1+m(\alpha) \sum_{i=1}^{r} k_{i}\left(s_{i}-1\right)\right),
$$

where $\zeta$ is the Dedekind zeta function of $F$, and define

$$
G_{\alpha}(s)=G_{m(\alpha)}\left(1 / 2+\sum_{i=1}^{r} k_{i}\left(s_{i}-1\right) / 2\right),
$$

where $G_{m}(s)$ is defined in (5.2). Finally we put

$$
Z^{*}(\mathbf{s} ; \mathbf{m}, \Psi)=Z(\mathbf{s} ; \mathbf{m}, \Psi) \prod_{\alpha>0} G_{\alpha}(s) \zeta_{\alpha}(s),
$$

and we can now state our main result: 
Theorem 6.1. Let $\Psi \in \mathfrak{M}(\Omega, \Phi)$. The function $Z(\mathbf{s} ; \mathbf{m}, \Psi)$ has a meromorphic continuation to $\mathbf{s} \in \mathbb{C}^{r}$. Moreover $Z(\mathbf{s} ; \mathbf{m}, \Psi)$ satisfies a group of functional equations under $W$, the Weyl group of $\Phi$. For the simple reflection $\sigma_{i}$ we have

$$
Z^{*}(\mathbf{s} ; \mathbf{m}, \Psi)=\left|m_{i}\right|^{1-s_{i}} Z^{*}\left(\sigma_{i} \mathbf{s} ; \mathbf{m}, \sigma_{i} \Psi\right) .
$$

The set of polar hyperplanes is contained in the $W$-translates of the hyperplanes $s_{i}=1 \pm 1 / \operatorname{gcd}\left(n,\left\|\alpha_{i}\right\|^{2}\right)$.

Proof. We will show that the functional equations are valid, whenever both sides of (6.2) are defined. Given this, the meromorphic continuation of $Z^{*}$ and the validity of the functional equations for all $\mathbf{s} \in \mathbb{C}^{r}$ away from the polar hyperplanes is a routine consequence of the Bochner's tube principle as in [CG07] or BBF06. We refer the reader to these papers for the details.

To prove the $\sigma_{i}$ functional equation, fix $c_{j}$ for $i \neq j$ and consider the sum over the $i$ th index in the series defining $Z(\mathbf{s} ; \mathbf{m}, \Psi)$ :

$$
\sum_{0 \neq c_{i} \in \mathcal{O}_{S} / \mathcal{O}_{S}^{\times}} \frac{H\left(c_{1}, \ldots, c_{r} ; \mathbf{m}\right) \Psi\left(c_{1}, \ldots, c_{r}\right)}{\left|c_{i}\right|^{s_{i}}} .
$$

This is the function $\mathcal{E}\left(s_{i}, \mathbf{c} ; \mathbf{m}, \Psi, i\right)$ of (5.9), which satisfies the functional equation of Theorem 5.8. The functional equation of $Z^{*}(\mathbf{s} ; \mathbf{m}, \Psi)$ under $\sigma_{i}$ is now immediate.

\section{EXAMPles AND COMMENTS}

We list some examples of the Weyl group multiple Dirichlet series constructed in this paper. In this section, we will simplify the exposition by ignoring such complications as the exact form of reciprocity, Hilbert symbols, and the use of $S$-integers instead of integers.

7.1. Quadratic series. In CG07] we gave a list of examples of quadratic Weyl group multiple Dirichlet series associated to simply-laced root systems. In summary, the $A_{2}, A_{3}$ and $D_{4}$ series can be used to study the first, second and third moments of quadratic Dirichlet $L$-functions, respectively, as in Goldfeld-Hoffstein GH85, Fisher-Friedberg [FF04, FF03] or Diaconu-Goldfeld-Hoffstein DGH03]. The firstnamed author used the $A_{5}$ quadratic series to study mean values of zeta functions of biquadratic fields in Chi05.

In BFH91, Bump, Friedberg, and Hoffstein computed the Whittaker coefficients of an Eisenstein series on the double cover of $G S p(2 r)$. The Eisenstein series they studied was induced from a (nonmetaplectic) cusp form on $G L(r)$. The authors found that the Whittaker coefficients of these Eisenstein series involved quadratic twists of the $L$-function of $f$. In particular, these Whittaker coefficients have Euler products. According to the Eisenstein conjecture stated in the introduction, when the cusp form $f$ is replaced by a minimal parabolic Eisenstein series on $G L(r)$, the Whittaker coefficients of the induced metaplectic Eisenstein series on the double cover of $G S p(2 r)$ are expected to coincide with the quadratic multiple Dirichlet series associated to the root system $B_{r}$. Though a Weyl group multiple Dirichlet series does not generally have an Euler product, it is easy to see from the relations (4.3),(4.4),(4.5) that for $n=2$ and root systems of type $B$ (normalized so that the short roots have length one), the function $H$ is actually multiplicative, not just twisted multiplicative. Therefore, modulo the validity of the Eisenstein conjecture, 
our formula (3.25) can be seen as an analogue of the Casselman-Shalika formula in this metaplectic context. Bump, Friedberg, and Hoffstein also give a CasselmanShalika formula in BFH91. In work in preparation, Brubaker, Bump, Chinta and Gunnells check that the formula of [BFH91] agrees with the formula of this paper for type $B$ and $n=2$.

7.2. Cubic series. When $n=3$, the Kubota Dirichlet series $\mathcal{D}(s, a)$ has the nice property that for $a \in \mathcal{O}_{S}$ squarefree, its residue at $s=4 / 3$ is, up to a constant and a power of $a$, the conjugate Gauss sum $\overline{g(1, a)}$ (cf. [Pat77a, Pat77b]). This was exploited by Brubaker and Bump BB06b] to show that residues of the $A_{3}, n=3$ series give two double Dirichlet series involving $L$-functions associated to cubic characters studied by Friedberg, Hoffstein, and Lieman [FHL03. Using similar reasoning, we expect that a triple residue of the $E_{6}, n=3$ series will give the multiple Dirichlet series in three variables studied by Brubaker Bru03. Labelling the outer nodes with the indices $1,2,3$, the $E_{6}$ series is heuristically of the form

$$
\sum_{a_{1}, \ldots, a_{6}} \frac{g\left(a_{4}, a_{1}\right) g\left(a_{5}, a_{2}\right) g\left(a_{6}, a_{3}\right) g\left(1, a_{4}\right) g\left(1, a_{5}\right) g\left(1, a_{6}\right) \overline{\left(\frac{a_{4}}{a_{5}}\right) \overline{\left(\frac{a_{6}}{a_{5}}\right)}}}{\left|a_{1}\right|^{s_{1}} \cdots\left|a_{6}\right|^{s_{6}}} .
$$

Taking residues in $s_{1}, s_{2}$ and $s_{3}$, the Gauss sums disappear and we expect to be left with a series of the form

$$
\sum_{a_{4}, a_{5}, a_{6}} \frac{\overline{\left(\frac{a_{4}}{a_{5}}\right)} \overline{\left(\frac{a_{6}}{a_{5}}\right)}}{\left|a_{4}\right|^{s_{4}^{\prime}}\left|a_{5}\right|^{s_{5}^{\prime}}\left|a_{6}\right|^{s_{6}^{\prime}}}
$$

for some new variables $s_{4}^{\prime}, s_{5}^{\prime}, s_{6}^{\prime}$. The squarefree coefficients coincide with those of a series used by Brubaker to prove an asymptotic formula for the second moment of a cubic Dirichlet $L$-series. Presumably, using the explicit description of the $\varpi$-part we have given in Section 3 and using the techniques of [BB06b] to compute the $\varpi$-parts of residues of the cubic series, we can show that residues of the $E_{6}, n=3$ series coincide with the series studied by Brubaker. We have not checked this in detail.

7.3. Higher $n$. Friedberg, Hoffstein, and Lieman [FHL03] have constructed double Dirichlet series built out of $L$-functions of order $n$ Hecke characters. As mentioned above, Brubaker and Bump show in [BB06b] that, when $n=3$, these series arise after taking a single residue of the $A_{3}, n=3$ series. They further conjecture that for general $n$, the series of [FHL03] are $(n-2)$-fold residues of the degree $n A_{n}$ series. The methods of [BB06b] do not work for $n>3$ because of our incomplete understanding of the residues of Kubota's Dirichlet series. However, exploiting the fact that a Weyl group multiple Dirichlet series over a function field can be explicitly computed as a rational function, J. Mohler has verified the conjecture of BB06b over the rational function field for $n \leq 9$. It is likely that his techniques will yield a proof for all $n$ in the setting of a rational function field.

7.4. The Weyl character formula. The construction of the rational function $h(\mathbf{x} ; \ell)$ in Theorem 3.5 suggests that it should be thought of as a deformation of the Weyl character formula. Indeed, it is not hard to prove

$$
\Delta(\mathbf{x})=\sum_{w \in W} j(w, \mathbf{x}) .
$$


With this result the definition of $h$ becomes

$$
h(\mathbf{x} ; \ell)=\frac{\sum_{w \in W} j(w, \mathbf{x})\left(\left.1\right|_{\ell} w\right)(\mathbf{x})}{\sum_{w \in W} j(w, \mathbf{x})},
$$

which is clearly analogous to the Weyl character formula.

More precisely, let $\mathfrak{g}$ be the complex semisimple Lie algebra determined by $\Phi$, and let $\omega_{1}, \ldots, \omega_{r}$ be the fundamental weights of $\Phi$. Write $h(\mathbf{x} ; \ell)=N(\mathbf{x} ; \ell) / D(\mathbf{x})$. If $n=1$, then it turns out that $N(\mathbf{x} ; \ell)$ is actually divisible by $D(\mathbf{x})$, so that $h(\mathbf{x} ; \ell)$ is actually a polynomial. After some simple changes of variables and introduction of $q$ powers, this polynomial can be identified with the character $\chi_{\theta}$ of the representation $V_{\theta}$ of $\mathfrak{g}$ of lowest weight $-\theta$, where as before $\theta=\sum\left(l_{i}+1\right) \omega_{i}$.

On the other hand, if $n>1$, then $N(\mathbf{x} ; \ell)$ is not divisible by $D(\mathbf{x})$ in general, and so $h$ is not a polynomial. Nevertheless, one still might view $h$ as a deformation of a character. We plan to explore this connection between characters, $N(\mathbf{x} ; \ell)$, and $h(\mathbf{x} ; \ell)$ in future work.

\section{ACKNOWLEDGMENTS}

The authors are deeply grateful to Ben Brubaker, Dan Bump, Sol Friedberg, Jeff Hoffstein, Joel Mohler, and Samuel Patterson for their advice and extensive correspondence throughout the preparation of this work. The authors also thank the referee for several comments that greatly improved our paper.

\section{REFERENCES}

[BB06a] Ben Brubaker and Daniel Bump. On Kubota's Dirichlet series. J. Reine Angew. Math., 598:159-184, 2006. MR2270571 (2007k:11139)

[BB06b] Ben Brubaker and Daniel Bump. Residues of Weyl group multiple Dirichlet series associated to $\widetilde{\mathrm{GL}}_{n+1}$. In Multiple Dirichlet series, automorphic forms, and analytic number theory, volume 75 of Proc. Sympos. Pure Math., pages 115-134. Amer. Math. Soc., Providence, RI, 2006. MR2279933 (2008h:11044)

$\left[\mathrm{BBC}^{+} 06\right]$ B. Brubaker, D. Bump, G. Chinta, S. Friedberg, and J. Hoffstein. Weyl group multiple Dirichlet series I. In S. Friedberg, D. Bump, D. Goldfeld, and J. Hoffstein, editors, Multiple Dirichlet Series, Automorphic Forms, and Analytic Number Theory, volume 75 of Proc. Sympos. Pure Math., pages 91-114, 2006. MR2279932(2007k:11073)

[BBFa] B. Brubaker, D. Bump, and S. Friedberg. Weyl group multiple Dirichlet series, Eisenstein series and crystal bases. Submitted.

[BBFb] B. Brubaker, D. Bump, and S. Friedberg. Weyl Group Multiple Dirichlet Series: Type A Combinatorial Theory. Submitted.

[BBF06] B. Brubaker, D. Bump, and S. Friedberg. Weyl group multiple Dirichlet series. II. The stable case. Invent. Math., 165(2):325-355, 2006. MR2231959 (2007g:11056)

[BBF08] Ben Brubaker, Daniel Bump, and Solomon Friedberg. Twisted Weyl group multiple Dirichlet series: The stable case. In Eisenstein series and applications, volume 258 of Progr. Math., pages 1-26. Birkhäuser Boston, Boston, MA, 2008. MR2402679

[BBFH07] B. Brubaker, D. Bump, S. Friedberg, and J. Hoffstein. Weyl group multiple Dirichlet series. III. Eisenstein series and twisted unstable $A_{r}$. Ann. of Math. (2), 166(1):293316, 2007. MR2342698

[BFH91] Daniel Bump, Solomon Friedberg, and Jeffrey Hoffstein. $p$-adic Whittaker functions on the metaplectic group. Duke Math. J., 63(2):379-397, 1991. MR.1115113 (92d:22024)

[Bru03] B. Brubaker. Analytic continuation for cubic multiple Dirichlet series. Thesis, Brown University, 2003.

[CFG08] Gautam Chinta, Solomon Friedberg, and Paul E. Gunnells. On the p-parts of quadratic Weyl group multiple Dirichlet series. J. Reine Angew. Math., 623:1-23, 2008. MR2458038 
[CFH06] Gautam Chinta, Solomon Friedberg, and Jeffrey Hoffstein. Multiple Dirichlet series and automorphic forms. In Multiple Dirichlet series, automorphic forms, and analytic number theory, volume 75 of Proc. Sympos. Pure Math., pages 3-41. Amer. Math. Soc., Providence, RI, 2006. MR2279929 (2008c:11072)

[CG] G. Chinta and P. E. Gunnells. Weyl group multiple Dirichlet series of type $A_{2}$. Submitted to the Lang memorial volume.

[CG07] G. Chinta and P. E. Gunnells. Weyl group multiple Dirichlet series constructed from quadratic characters. Invent. Math., 167(2):327-353, 2007. MR2270457(2007j:11116)

[Chi05] G. Chinta. Mean values of biquadratic zeta functions. Invent. Math., 160(1):145-163, 2005. MR2129710(2006a:11108)

[Chi08] G. Chinta. Multiple Dirichlet series over rational function fields. Acta Arith., 132(4):377-391, 2008. MR.2413360 (2009e:11175)

[DGH03] Adrian Diaconu, Dorian Goldfeld, and Jeffrey Hoffstein. Multiple Dirichlet series and moments of zeta and L-functions. Compositio Math., 139(3):297-360, 2003. MR2041614(2005a:11124)

[FF03] Benji Fisher and Solomon Friedberg. Sums of twisted GL(2) $L$-functions over function fields. Duke Math. J., 117(3):543-570, 2003. MR.1979053 (2004c:11072)

[FF04] Benji Fisher and Solomon Friedberg. Double Dirichlet series over function fields. Compos. Math., 140(3):613-630, 2004. MR2041772 (2005a:11183)

[FHL03] Solomon Friedberg, Jeffrey Hoffstein, and Daniel Lieman. Double Dirichlet series and the $n$-th order twists of Hecke $L$-series. Math. Ann., 327(2):315-338, 2003. MR2015073 (2005b:11182)

[GH85] Dorian Goldfeld and Jeffrey Hoffstein. Eisenstein series of $\frac{1}{2}$-integral weight and the mean value of real Dirichlet $L$-series. Invent. Math., 80(2):185-208, 1985. MR788407 (86m:11029)

[Hof92] Jeffrey Hoffstein. Theta functions on the $n$-fold metaplectic cover of SL(2) - the function field case. Invent. Math., 107(1):61-86, 1992. MR.1135464(92k:11049)

[Hum90] James E. Humphreys. Reflection groups and Coxeter groups, volume 29 of Cambridge Studies in Advanced Mathematics. Cambridge University Press, Cambridge, 1990. MR:1066460 (92h:20002)

[IR90] Kenneth Ireland and Michael Rosen. A classical introduction to modern number theory, volume 84 of Graduate Texts in Mathematics. Springer-Verlag, New York, second edition, 1990. MR.1070716 (92e:11001)

[KP84] D. A. Kazhdan and S. J. Patterson. Metaplectic forms. Inst. Hautes Études Sci. Publ. Math., (59):35-142, 1984. MR743816 (85g:22033)

[Kub71a] Tomio Kubota. Some number-theoretical results on real analytic automorphic forms. In Several Complex Variables, II (Proc. Internat. Conf., Univ. Maryland, College Park, Md., 1970), pages 87-96. Lecture Notes in Math., Vol. 185. Springer, Berlin, 1971. MR0314768 (47:3320)

[Kub71b] Tomio Kubota. Some results concerning reciprocity law and real analytic automorphic functions. In 1969 Number Theory Institute (Proc. Sympos. Pure Math., Vol. XX, State Univ. New York, Stony Brook, N.Y., 1969), pages 382-395. Amer. Math. Soc., Providence, R.I., 1971. MR0340221 (49:4976)

[Neu99] Jürgen Neukirch. Algebraic number theory, volume 322 of Grundlehren der Mathematischen Wissenschaften [Fundamental Principles of Mathematical Sciences]. SpringerVerlag, Berlin, 1999. Translated from the 1992 German original and with a note by Norbert Schappacher, With a foreword by G. Harder. MR.1697859 (2000m:11104)

[Pat77a] S. J. Patterson. A cubic analogue of the theta series. J. Reine Angew. Math., 296:125161, 1977. MR0563068 (58:27795a)

[Pat77b] S. J. Patterson. A cubic analogue of the theta series. II. J. Reine Angew. Math., 296:217-220, 1977. MR0563069 (58:27795b)

[Pat07] S. J. Patterson. Note on a paper of J. Hoffstein. Glasg. Math. J., 49(2):243-255, 2007. MR2347258(2008h:11039) 
Department of Mathematics, The City College of CUNy, New York, New York 10031

E-mail address: chinta@sci.ccny.cuny.edu

Department of Mathematics and Statistics, University of Massachusetts, Amherst, MASSACHUSETTS 01003

E-mail address: gunnells@math.umass.edu 